\title{
ANALYSIS OF IMPACT OF MACHINE-TYPE-COMMUNICATION ON HUMAN-TYPE COMMUNICATION OVER WIRELESS COMMUNICATION NETWORKS
}

\author{
by \\ Parampreet Sidhu \\ BCA, Guru Nanak Dev University, Amritsar, India, 2008
}

\author{
A thesis \\ presented to Ryerson University \\ in partial fulfilment of the \\ requirements for the degree of \\ Master of Science \\ in the Program of \\ Computer Science
}

Toronto, Ontario, Canada, 2015

(C)Parampreet Sidhu 2015 


\section{AUTHOR'S DECLARATION FOR ELECTRONIC SUBMISSION OF A THESIS}

I hereby declare that I am the sole author of this thesis. This is a true copy of the thesis, including any required final revisions, as accepted by my examiners.

I authorize Ryerson University to lend this thesis to other institutions or individuals for the purpose of scholarly research.

I further authorize Ryerson University to reproduce this thesis by photocopying or by other means, in total or in part, at the request of other institutions or individuals for the purpose of scholarly research.

I understand that my thesis may be made electronically available to the public. 


\title{
ANALYSIS OF IMPACT OF MACHINE-TYPE-COMMUNICATION ON HUMAN-TYPE COMMUNICATION OVER WIRELESS COMMUNICATION NETWORKS
}

\author{
Parampreet Sidhu \\ MSc, Computer Science, Ryerson University, 2015
}

\begin{abstract}
With the advent of new wireless technologies, it is expected that the use of MachineType Communication (MTC) will significantly increase in next generation wireless networks. Wireless communication networks are considered to support MTC due to their availability and existing infrastructures. As these networks are designed and optimized in a way that they fit best for Human Type Communication (HTC), there is a need of an efficient radio resource management (RRM) to accommodate MTC traffic without affecting the regular HTC traffic in the network. In this thesis, a continuous-time Markov chain (CTMC) model-based RRM scheme is proposed to analyze the impact of MTC traffic on HTC traffic in wireless communication networks, in terms of blocking probability and channel utilization. Numerical results are provided, demonstrating the effectiveness of the proposed RRM scheme in providing the quality of service (QoS) isolation between HTC and MTC traffic.
\end{abstract}




\section{Acknowledgments}

I would like to express my gratitude to each and everyone who supported me throughout my journey in Ryerson University. My special thanks to my supervisor Dr. Isaac Woungang, and my co-supervisor, Dr. Glaucio H. S. Carvalho, for their continuous support, patience, motivation, enthusiasm and time. I feel privileged to study under their guidance. I am also thankful to the Department of Computer Science and the School of Graduate Studies at Ryerson University for all the financial and infrastructural assistance.

My gratitude also goes to my husband for his motivation and ever ready attitude for

help. A special thanks to my parents for their support. Last but not least, thanks to all my contemporaries for being there in time of needs. 


\section{Contents}

List of Tables $\quad$ viii

List of Figures $\quad$ ix

List of Abbreviations $\quad$ xi

1 Introduction $\quad 1$

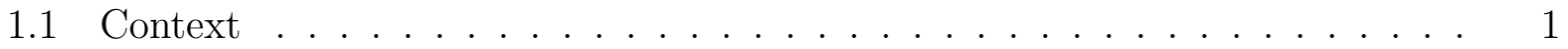

1.2 Research Problem . . . . . . . . . . . . . . . . . . . . 3

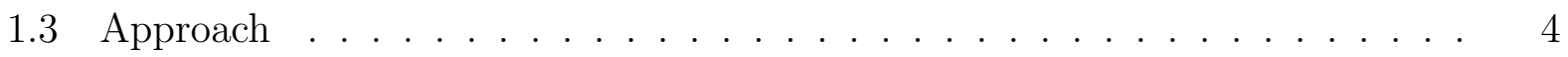

1.4 Thesis Contributions . . . . . . . . . . . . . . . . . 4

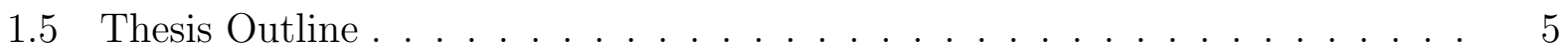

2 Background and Related Works $\quad 6$

2.1 Background ........................... 6

2.1.1 Wireless Communication Networks . . . . . . . . . . . . 6

2.1.2 Human-Type Communication . . . . . . . . . . . . . . . 9

2.1.3 Machine-Type Communication . . . . . . . . . . . . . 11

2.1.4 Current State-Of-The-Art in 3GPP Specifications . . . . . . . . . . 13

2.1.5 Radio Resource Management . . . . . . . . . . . . . . . . . 17

2.2 Related Work . . . . . . . . . . . . . . . . . . . . 19 
3 Proposed Radio Resource Management Scheme

3.1 System Model and Traffic Assumptions . . . . . . . . . . . . . . . . 24

3.2 CTMC-Based RRM Formulation . . . . . . . . . . . . . . . . . . 27

3.2.1 States of the CTMC Model . . . . . . . . . . . . . . 27

3.2 .2 States Transition . . . . . . . . . . . . . . . . 27

3.2.3 Blocking Probabilities and Channel Utilization . . . . . . . . . . . . 29

4 Performance Evaluation $\quad 32$

4.1 Network Parameters . . . . . . . . . . . . . . . . . 32

4.2 Scenarios Under Analysis . . . . . . . . . . . . . . . . . . . . 33

4.3 Performance Metrics . . . . . . . . . . . . . . . . . . . 33

4.4 Numerical Results . . . . . . . . . . . . . . . . . . . . 34

4.4.1 Scenario I: Impact of the Variation of the Arrival Rate of MTC traffic on the Blocking Probability of HTC Traffic . . . . . . . . . . . . . 34

4.4.2 Scenario II: Impact of the Variation of the Arrival Rate of MTC Traffic on the Blocking Probability of MTC Traffic . . . . . . . . . . . . 38

4.4.3 Scenario III: Impact of the Variation of the Arrival Rate of MTC traffic on the Channel Utilization for HTC traffic . . . . . . . . . . . . 41

4.4.4 Scenario IV: Impact of the Variation of the Arrival Rate of MTC traffic on the Channel Utilization for MTC traffic . . . . . . . . . . . . . . 43

4.4.5 Scenario V: Impact of the Variation of the Arrival Rate of MTC traffic on the Channel Utilization for the Shared Area . . . . . . . . . . 45

4.4.6 Scenario VI: Impact of the Variation of the Arrival Rate of HTC traffic on the Channel Utilization for the Shared Area . . . . . . . . . . . 48

5 Conclusion $\quad 53$

$\begin{array}{ll}\text { A Pseudocode for CTMC model } & 56\end{array}$ 
Bibliography 


\section{List of Tables}

2.1 Types of HTC traffic in cellular network . . . . . . . . . . . . . 10

2.2 MTC applications . . . . . . . . . . . . . . . . . . . . . . 14

3.1 State transitions . . . . . . . . . . . . . . . . . . . . . . 28

4.1 Network parameters. . . . . . . . . . . . . . . . . . . . . . . . . . 32

4.2 Threshold values. . . . . . . . . . . . . . . . . . . . . 34 


\section{List of Figures}

2.1 Evolution of cellular networks in terms of data rates . . . . . . . . . 8

2.2 Generic architecture of MTC . . . . . . . . . . . . . . . . 15

2.3 MTC Devices communicating directly with each other. . . . . . . . . . 16

2.4 MTC Devices communicating with one or more MTC servers (a) MTC Server located inside the network domain and (b) MTC Server located outside the network domain. . . . . . . . . . . . . . . . . . . . 16

3.1 RRM scheme for HTC and MTC traffic. . . . . . . . . . . . . . 25

3.2 Flowchart of the proposed RRM scheme for HTC and MTC traffic. . . . . 26

3.3 State transition diagram of state s of the CTMC model (a) Transitions to state $s=(1,1,1,1) \in \mathrm{S}$ and (b) Transitions from state $s=(1,1,1,1) \in \mathrm{S} . \quad . \quad 29$

4.1 Blocking probability of HTC vs. arrival rate of MTC with varied thresholds. 35

4.2 Blocking probability of HTC vs. arrival rate of MTC with constant MTC threshold. . . . . . . . . . . . . . . . . . . . 36

4.3 Blocking probability of HTC vs. arrival rate of MTC with constant HTC

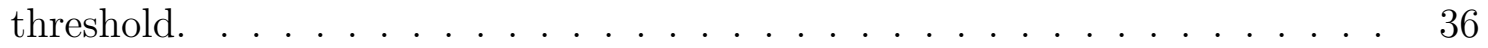

4.4 Blocking probability of HTC vs. arrival rate of MTC without thresholds. . . 37

4.5 Blocking probability of MTC vs. arrival rate of MTC with varied thresholds. 38

4.6 Blocking probability of MTC vs. arrival rate of MTC with constant MTC threshold. . . . . . . . . . . . . . . . . . . . . . . . . 39 
4.7 Blocking probability of MTC vs. arrival rate of MTC with constant HTC threshold. ......................... . . . 40

4.8 Blocking probability of MTC vs. arrival rate of MTC without thresholds. . . 41

4.9 Channel Utilization for HTC vs. arrival rate of MTC with varied thresholds. 42

4.10 Channel Utilization for HTC vs. arrival rate of MTC with constant MTC threshold. . . . . . . . . . . . . . . . . . . . 42

4.11 Channel Utilization for HTC vs. arrival rate of MTC with constant HTC threshold. . . . . . . . . . . . . . . . . . . . . 43

4.12 Channel Utilization for MTC vs. arrival rate of MTC with varied thresholds. 44

4.13 Channel Utilization for MTC vs. arrival rate of MTC with constant MTC threshold. . . . . . . . . . . . . . . . . . . . . 44

4.14 Channel Utilization for MTC vs. arrival rate of MTC with constant HTC threshold. . . . . . . . . . . . . . . . . . . 45

4.15 Channel Utilization of shared area vs. arrival rate of MTC with varied thresholds. . . . . . . . . . . . . . . . . . . . . . 46

4.16 Channel Utilization of shared area vs. arrival rate of MTC with constant MTC threshold. . . . . . . . . . . . . . . . . . . . . 47

4.17 Channel Utilization of shared area vs. arrival rate of MTC with constant HTC threshold. . . . . . . . . . . . . . . . . . . . . . 47

4.18 Channel Utilization of shared area vs. arrival rate of MTC without thresholds. 48

4.19 Channel Utilization of shared area vs. arrival rate of HTC with varied thresholds.

4.20 Channel Utilization of shared area vs. arrival rate of HTC with constant MTC threshold.

4.21 Channel Utilization of shared area vs. arrival rate of HTC with constant HTC threshold. . . . . . . . . . . . . . . . . . . . . . 51

4.22 Channel Utilization of shared area vs. arrival rate of HTC without thresholds. 51 


\section{List of Abbreviations}

- 1G: First Generation Telecommunication

- 2G: Second Generation Telecommunication

- 3G: Third Generation Telecommunication

- 3GPP: Third Generation Partnership Project

- 4G: Fourth Generation Telecommunication

- API: Application Programming Interface

- AT\&T: American Telephone \& Telegraph

- BS: Base Station

- CABM: Context-Aware Backhaul Management

- CDMA: Code Division Multiple Access

- CTMC: Continuous-Time Markov Chain

- ECACB: Enhanced Cooperative Access Class Barring

- EDGE: Enhanced Data rates for GSM Evolution

- GPRS: General Packet Radio Service

- GSM: Global System for Mobile Communications

- HSPA: High Speed Packet Access

- HTC: Human Type Communication

- IoT: Internet of Things 
- IP: Internet Protocol

- LANs: Local Area Networks

- LTE: Long-Term Evolution

- M2M: Machine-to-Machine

- MNO: Mobile Network Operators

- MTC: Machine Type Communication

- MTS: Mobile Telephone Service

- NMT: Nordic Mobile Telephone

- NTT: Nippon Telegraph and Telephone

- ORA: Orthogonal Resource Allocation

- QoS: Quality of Service

- PAYD: Pay As You Drive

- RANs: Radio Access Networks

- RCE: Recursive Contending Users Estimation

- RRM : Radio Resource Management

- RUPRA: Random User-Pairing Resource Allocation

- SA2: System Architecture Working Group 2

- SMRA: Suboptimal Minimal Resource Allocation

- SMS: Short Message Service

- TARRM: Traffic Adaptive Radio Resource Management 
- UE: User Equipment

- UMTS: Universal Mobile Telecommunications System

- VoIP: Voice over Internet Protocol

- WANs: Wide Area Networks

- WCDMA: Wideband Code Division Multiple Access

- Wi-Fi: Wireless Fidelity

- WiMax: Worldwide Interoperability for Microwave Access 


\section{Chapter 1}

\section{Introduction}

\subsection{Context}

The last few decades have been in full agreement with Moore's Law [1] which states that computing hardware will double its capacity every two years. The increase in computing power and size of devices has decreased by many folds during this time. As computing devices have grown toward compactness, communication networks have grown toward wireless. Future world is going to be comparatively free from wired networks and related infrastructures. Wireless networks have already begin to replaced the major chunk of wired networks. Wireless LANs/WANs, mesh networks, and cellular networks have been used vastly to support wide variety of needs in business and personal areas. In the last decade, cellular networks have emerged as the biggest area of implementation. They have grown at rapid speed, covering most parts of the world and they are supposed to grow more to serve the ever increasing demands. Wireless infrastructure used in cellular communication was initially optimized to meet the human type communication (HTC) or voice data. But with the emerging growth of the Internet of Things (IoT) [2] and Machine Type Communication (MTC), it became the contender in other types of usages over the period of time. The benefits of wireless cellular networks include availability in diverse geographical areas and cost factor, which 
may help their deployment in a variety of applications. Today, these wireless networks are used starting from simple telephone calls to voice over internet protocol (VoIP) traffic, from simple data packet transfer $(2 \mathrm{G})$ to complex video streaming (LTE), from transferring the meter readings to transmitting the live health data of patients, to name a few. The current world usage of cellular networks is vast and the future potentials of its untapped power are tremendous.

On the other hand, the remarkable growth in capabilities of computing machines has made it possible for gadgets to interact with each other directly or with minimal or no human intervention. This gave birth to MTC or Machine to Machine Communications (M2M) [3]. MTC refers to allowing direct communications between MTC devices or from MTC devices to one or more central MTC-based servers [3], using wired or wireless networks. In a small span of time, MTC devices found their usage in smart meters, intelligent transportation systems, tracking and tracing gadgets, health sector (via telehealth services), security sector (via the use of automated audio-visual monitoring) industrial wireless automation, ambient assisted living, to name a few [3]. A rapid growth is observed in the design of MTC and its applications. At the same time, it is projected [4] that by the end of this decade, there will be over millions of MTC devices and connections in service.

The major component of the MTC landscape is the communication method being used to transfer the information from one device to another. There is a vast scope of study and improvement concealed in the MTC framework [3]. With such a potential of applications and growth, the medium of communication between MTC-enabled devices takes the central stage. The characteristics of MTC include smaller packet sizes and frequent transmission. The quality of service (QoS) also plays an important role in some applications of MTC devices such as health care monitoring. One of the most prominent ways to create a communication channel for MTC devices is to setup a completely optimized wireless network that will service the MTC traffic. But designing such a network may necessitate a huge amount of cost and resources. Another alternative way is to use the existing wired networks. But 
this approach will definitely limit the usage to compact geographical areas only. Thus, existing wireless cellular networks appear as the best possible solution. It has been advocated in [5] existing wireless networks can be used for combined HTC and MTC traffic. But, incorporating the MTC devices into the existing HTC oriented cellular networks will bring its own set of challenges, for instance, in terms of adjusting the Third Generation Partnership Project (3GPP) standards [5]. However, in doing so, some challenging issues such as resource management and QoS degradation have to be addressed.

The complexity of using the existing wireless infrastructures for MTC in an environment that was optimized for HTC usage has opened up the door for a wide area of investigations. In [6], Liu et al. discussed the importance of categorizing the QoS of MTC and HTC traffic in cellular networks. In [7], Makris et al. focused on the study of the resource management for combined HTC and MTC traffic, with the goal to provide desired QoS to both traffic types. In [8], Wei et al. studied the problem of radio access networks overload, resulting from a mass access to the network by MTC devices, which may degrade the service quality of HTC. In this thesis, we propose a radio resource management (RRM) scheme for analyzing the influence of MTC traffic on HTC traffic over wireless communication networks.

\subsection{Research Problem}

In wireless communication networks, a RRM plays a critical role for the maximum utilization of the available resources. An efficient RRM scheme can significantly reduce the hardware requirements. Due to the deployed infrastructure and expanded coverage, cellular networks are best suited for the implementation of MTC applications since they can serve as a hub or backbone of the MTC implementation environment. MTC has diverse QoS requirements and features such as small packet size, frequent transmissions, large number of devices, to name a few, which are different from the regular HTC traffic of cellular networks in

terms of traffic characteristics. These differences lead to the key challenge of assigning 
the resources efficiently in an environment of combined HTC and MTC traffic in cellular networks. In fact, designing an efficient RRM scheme requires that the impact of MTC traffic on HTC traffic be analyzed in-depth, i.e. the performance of HTC traffic in the presence of a large number of MTC devices in the network has to be investigated. In this thesis, the problem of degradation in the performance of HTC due to the presence of MTC traffic in cellular networks is addressed, and the impact of MTC traffic on HTC traffic in wireless communication networks is analyzed by means of a novel RRM scheme.

\subsection{Approach}

A continuous-time Markov chain (CTMC) model is utilized to formulate the RRM scheme that supports two different types of service requests, namely HTC and MTC. The proposed RRM scheme allows to analyze the performance of the MTC and HTC integration over the air interface. In its design, the radio resources are distributed between MTC traffic, HTC traffic, and an area shared by both the MTC and HTC traffic. Two thresholds are setup to distribute the radio channels in a predefined way. The system will accept the HTC or MTC service request as long as their dedicated radio channels are available. Once the HTC or MTC service requests that are already in the system have reached their predefined thresholds, the system will forward any new incoming HTC or MTC request to the shared area if it has some resources available. Otherwise, the incoming HTC or MTC incoming service request will be rejected. In Chapter 4, the performance of the proposed RRM scheme is evaluated analytically under varying threshold values, using both service types, with the goal to study the impact of MTC traffic over HTC traffic in terms of blocking probability and channel utilization.

\subsection{Thesis Contributions}

The main contributions of this thesis are: 
- Design of a novel CTMC-based RRM scheme for wireless communication networks.

- Evaluation of the proposed RRM scheme through an analysis of the MTC on HTC over wireless communication networks.

\subsection{Thesis Outline}

The remainder of this thesis is organized as follows:

- Chapter 2 presents some background information on wireless networks and radio resource allocation for MTC and HTC traffic.

- Chapter 3 describes the proposed CTMC-based RRM scheme, along with the system model and traffic assumptions.

- Chapter 4 describes the performance evaluation of the proposed CTMC-based RRM scheme.

- Chapter 5 concludes our work and highlights some future work. 


\section{Chapter 2}

\section{Background and Related Works}

\subsection{Background}

The objective of this chapter is to shed some light on the background of wireless networks from a communication technology perspective. A brief history of cellular networks and its improvements over the past decade are discussed. The impact of these advancements on the next generation of devices such as MTC-based devices is briefly discussed. As HTC traffic constitutes the base of cellular networks, its characteristics and main applications are discussed. The characteristics and applications of MTC along with its current state in 3GPP are also described. The usage of MTC over the existing HTC traffic in cellular networks has resulted to new challenges and complexity in terms of radio resource management (RRM), which are also discussed.

\subsubsection{Wireless Communication Networks}

In the current era, various types of wireless networks are being used to transfer the data between different types of devices in wireless communication networks. Examples of such networks include wireless LAN, Wi-Fi, Wi-Max, ZigBee, TransferJet, Bluetooth, Ham radio network, cellular networks, to name a few. Among these, cellular networks are the most 
widely used networks because they have been proven to cover a wide range of geographical areas. The technology of these networks has evolved from GSM to 2G, 3G and nowadays 4G or LTE [9]. This technology evolution has been made possible due to noticeable advancements in human-centric computing, microarchitecture design, power-performance of multi-threaded and multi-core processors, improved battery/power life, digital signal processing, transmitters design, just to name a few. Throughout its evolving cycles, cellular networks have continuously provided better data rates. In the initial starting phase of $2 \mathrm{G}-$ based cellular networks, a data rate of $14.4 \mathrm{kbps}$ was achieved, and improved over time to 171 kpbs by using the General Packet Radio Service (GPRS) technology, to 384 kbps by using the Enhanced Data Rates for GSM Evolution (EDGE) technology, to 2 Mbps during the 3G phase using the Wideband Code Division Multiple Access (WCDMA) technology, to 14.4 Mbps using the High Speed Packet Access (HSPA) technology, and nowadays to the range 50-100 Mbps along with guaranteed QoS, improved spectrum efficiency and larger coverage using the 4G (or LTE) technology [9]. This evolution of cellular networks technology in terms of data rates is shown in Fig. 2.1.

Cellular networks work and expand with the basic building blocks referred to as cells. Cells define the coverage area of a cellular network. These cells are served by an infrastructure known as Base Station (BS) or collection of BSs. The physical devices such as antenna, power backups, signal transmitters, processing devices, to name a few, are located in these BSs. The area covered by the cell depends on the capacity of the BSs, which are themselves connected to the core network and are assigned a group of radio frequency bands or channels. Each BS can support multiple users or devices which are connected to it by means of a range of radio frequencies or channels. Due to the rules and regulations that were put in place by governments and technology standard bodies, only a specific set of frequencies can be used to transmit the mobile signals. This constraint indeed limits the number of radio channels which can be used in BSs to transmit the signals. Since the channels are limited and there are multiple users and cells in the network, setting up the parameters such as data rates, user 


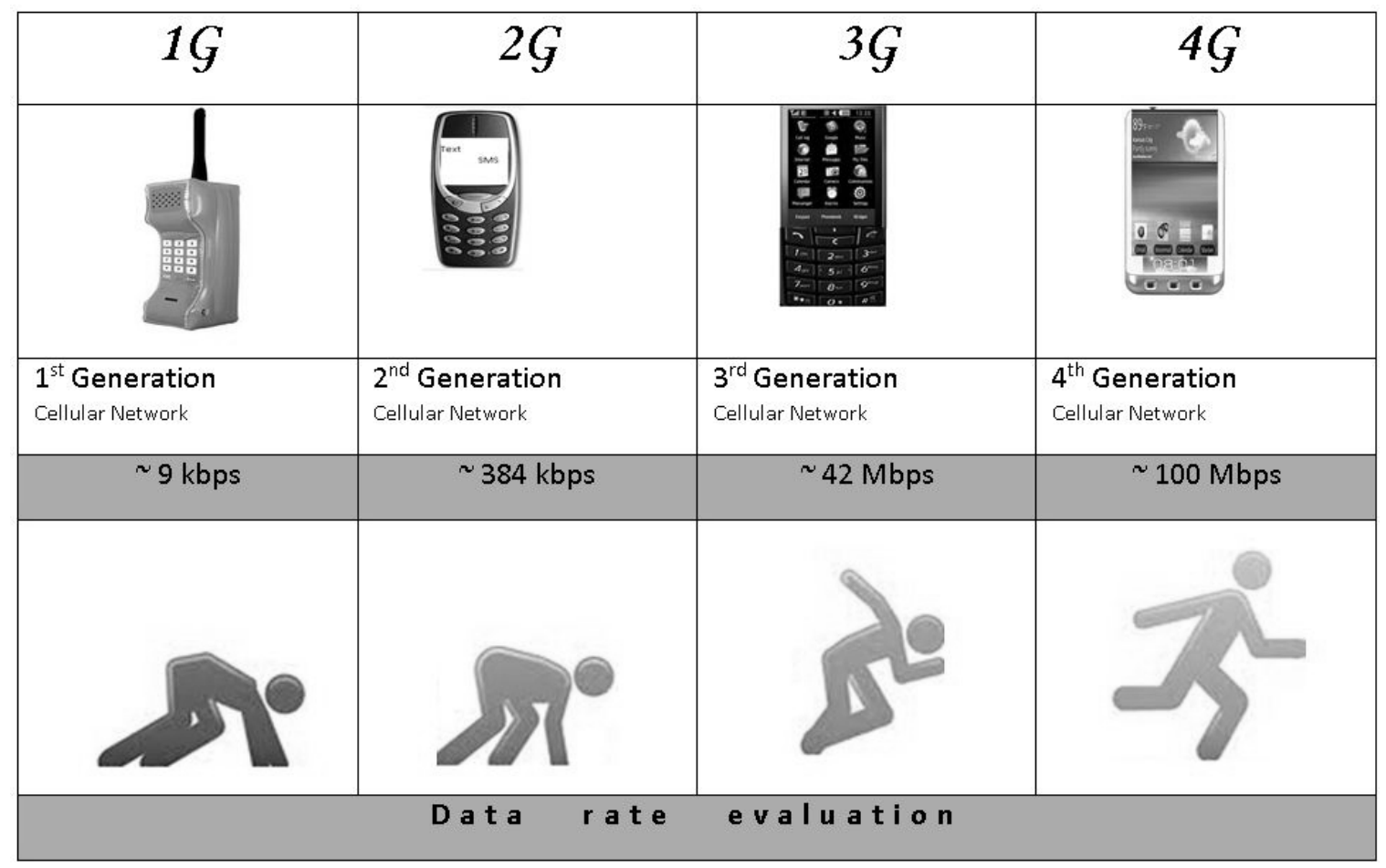

Figure 2.1: Evolution of cellular networks in terms of data rates

allocations, transmission power and receiving power, modulation scheme, handover criteria, to name a few, play an important role in the management of the resources.

Due to advances in wireless technologies, wireless cellular networks, which were initially optimized to transfer the voice data, are now transitioning from the mobile phones age to the wireless computing age, resulting to an increase in data transfer rates. This allows cellular networks to provide services such as video streaming. With this new development, all the communications that were originally initiated with human intervention via mobile phones or similar devices using HTC still prevail, in addition to allowing the devices to enable communication between each other without human intervention thanks to advances in hardware technology, computational power of devices, and artificial intelligence. This improvement has opened the door for future technologies such MTC to extend the features of the established cellular networks. Cellular networks have tremendous untapped potentials which can change the lifestyle of our next generations by providing far more services like 
wireless TV, security monitoring, tele-medicine, tracking and tracing, to name a few.

\subsubsection{Human-Type Communication}

Although mobile services started a while ago when AT\&T commercialized the Mobile Telephone Service (MTS) in mid 1940s [10], the first handheld cellular phone was developed by Motorola in 1973 [11]. The cellular networks design arisen in the late 1970s and early 1980s with the deployment of automatic analog cellular systems by NTT in Tokyo (1979) and by NMT in Nordic countries (1981) [10]. This was the beginning of HTC with the first generation $(1 \mathrm{G})$ of cellular networks, where analog signals were used to transmit the information. The network traffic was referred to as HTC because of the human intervention required for initiating and accepting the calls (phone calls only). The second generation (2G) of cellular networks has arisen from the digitization of signals and the addition of new features such as SMS. In parallel, Global System for Mobile Communications (GSM) and Code Division Multiple Access (CDMA) have also emerged as the standards using digital signals for transmission instead of analog signals, which help improving the quality of the calls for end users. With these technologies, 2G-based cellular networks were able to support basic media such as ringtones via mobile devices, as well as basic Internet services such as browsing, email transfers, but at low data rates. They were also optimized to support HTC only.

The introduction of basic internet services and the quality of services provided by $2 \mathrm{G}$ based cellular networks attracted a large number of consumers to use these networks. Due to the increasing demand for high data rates, 2G-based cellular networks rapidly moved to 3G-based cellular networks via the implementation of the High Speed Packet Access (HSPA) mechanism over the Universal Mobile Telecommunications System (UMTS)-based networks. With this enhancement, applications over 3G-based cellular networks such as calls, browsing, streaming, video calls, file transfer, to name a few, still remain human centric and human interactions were still required for their initiations, acceptance and actions. With this increase in the number of users and limited resources, QoS has become the main concern for 
incoming HTC traffic in 3G-based cellular networks. To handle the traffic efficiently, HTC services should be mapped into four classes, namely: conversational, streaming, interactive and background [12]. With this categorization, an important QoS parameter which can be used to segregate the services into different class is the delay sensitivity (i.e. time delay parameter which is often used in multimedia applications). Services such video call and delivery of email have different impact on the end users as far as this delay factor is concerned. A delay of 5 seconds in video call matters far more than a delay of 5 seconds in email transfers. Most delay sensitive applications such as video calls and live streaming belong to the conversational class whereas least delay sensitive applications such as file download are kept in the background class. Both conversational and streaming classes handle real time traffic to give more responsive network to end users whereas interactive and background classes support basic internet applications such as browsing, email transfer, to name a few $[12]$.

Cellular networks are moving progressively from voice centric systems to data centric systems and the current generation of wireless networks (referred to as $4 \mathrm{G}$ systems) support other types of data centric traffics such as MTC along with HTC traffic. In the future 5G systems, the use of MTC on HTC over cellular networks (as communication backbone) is expected to grow significantly. Table 2.1 shows the traffic types of HTC in cellular networks.

\begin{tabular}{||c|c||}
\hline \hline Generations & HTC traffic type \\
\hline \hline $1 \mathrm{G}$ & Voice (Mobile call) \\
\hline $2 \mathrm{G}$ & Digital Voice \\
& SMS \\
& Data (low data rate) \\
\hline $3 \mathrm{G}$ & $\begin{array}{c}\text { High quality audio, graphics and video } \\
\text { Data }\end{array}$ \\
\hline $4 \mathrm{G}$ & High speed data \\
& VoIP \\
\hline \hline
\end{tabular}

Table 2.1: Types of HTC traffic in cellular network 


\subsubsection{Machine-Type Communication}

MTC or Machine-to-Machine communication (M2M) can be defined as a form of data communication where two or more entities interact independently with each other without any human interactions or supervision. This communication can either happen using the wired or wireless systems. The main idea of MTC is to reduce the dependency of devices over human actions, making them self-sufficient to initiate the actions based on the available network information. In order to replace the decision making intelligence of human with that of machines, it is required that some information be gathered from the devices, including the devices processing power. Typically, a large number of MTC devices are involved in MTC applications, and in most cases, MTC devices support the uplink transmission of data. MTC applications include but are not limited to transportation, health care, safety, security, tracking, home automation, to name a few, and cellular networks are suited for these types of applications.

Some important benefits of using MTC in cellular networks include: (1) the use of the vast geographical coverage area provided by the cellular networks. Indeed, using MTC in cellular networks, mobile network operators (MNOs) can provide seamless cellular services all over the world with robust security solutions, high mobility, delay guarantees, high bandwidth, with limited changes in the current standards and low cost of implementation and operations; (2) Cellular networks technologies (such as femtocell) [13] can be used to provide the desired QoS for critical applications such as tele-medecine, old age care homes, to name a few.

A rapid growth in the use of MTC devices in cellular networks is expected to occur in the next decade, with an annual rate of more than $20 \%$ to reach 200 million MTC devices till 2019 [4]. According to GSMA [14], a leading pan-european organization, MTC connections have reached 195 million at the end of 2013 and about 250 million connections are expected to be reached by early 2015 .

Salient features of MTC applications (different from those of HTC applications) have been described in the 3rd Generation Partnership (3GPP) project draft [15]. It is reported 
that it is not necessary for an MTC application to follow strictly all the features of HTC applications, and these features can be activated individually in a system. The features of MTC as defined by 3GPP Release 10 [5] can be summarized as follows:

- Small data transmissions: small data packets can be exchanged in MTC traffic. In addition, MTC devices can send the recorded data such as temperature, meter readings, GPS coordinate, to name a few.

- Large number of devices: MTC traffic can be associated with a large number of devices connected to a network at the same time.

- Low mobility: the movement of MTC devices is very limited, and in general, is restricted to a certain predefined area only.

- Time controlled: the transmission and receipt of data by MTC devices are restricted to particular time intervals (slots).

- Time tolerance: MTC devices can sense the traffic and can delay their data transmission.

- Priority alarm: MTC devices can send priority alarm messages such as theft alert, fire alert, to name a few.

- Packet switched only: packet switched services are provided to MTC devices with or without the need to allocate a mobile subscriber integrated services digital network number.

- Secure connection: a secure connection is required between MTC devices and servers.

- MTC monitoring: this feature is used by MTC applications that require the monitoring of the events related to MTC devices.

- Location specific trigger: MTC devices are triggered by using their location information. 
- Infrequent transmission: random transmission and long intervals between consecutive transmissions from MTC devices can be implemented.

- Mobile originated communication only: mobile originated communication can be implemented for mobile MTC applications that require this feature.

- Infrequent mobility termination: This feature is used to reduce the mobility management frequency of MTC devices that support mobile originated communications.

- Network provided destination for uplink data: this feature can be used for the purpose of uplink transmission of data to the network.

- Group-based MTC features: MTC devices can be managed as a group in case the same message needs to be transmitted or a combined QoS policy needs to be enforced on multiple MTC devices.

A variety of MTC-based applications have been reported in public and private sectors, some of which are captured in Table 2.2 .

\subsubsection{Current State-Of-The-Art in 3GPP Specifications}

The initial study on MTC by 3GPP was introduced in its Release 8 [3]. In 3GPP release 10 [5], the support for MTC traffic along with the service requirements for MTC traffic were introduced. These include the subscription options, the process of sending and receiving the data based on triggers, the addressing schemes, the charging, security, and remote management requirements, to name a few. The system architecture Working Group 2 (SA2) of 3GPP defined the architectural requirements and models to support MTC in 3GPP networks [16]. In the future 3GPP release 10+ [16], it is expected that significant efforts will be dedicated to analyzing and optimizing the network architecture for the purpose of reducing the impact of MTC on the regular traffic in cellular networks. In this context, due to the expected use of a large number of MTC devices, key issues such as IP addressing, signalling 


\begin{tabular}{||c|c||}
\hline \hline MTC Applications & Examples \\
\hline \hline Tracking and Tracing & Emergency call \\
& Fleet management \\
& Theft Tracking \\
& Traffic Information \\
& Navigation \\
& Pay as you drive (PAYD) \\
\hline Smart Meters & Electricity \\
& Gas \\
& Water \\
\hline Health & Remote patient monitoring \\
& Assisted living \\
& Personal fitness \\
\hline Security & Access control \\
& Alarm Systems \\
& Surveillance systems \\
\hline Home Automation & Thermostat control \\
& Lighting control \\
& Appliance control \\
\hline Remote Maintenance and Control & Vehicle diagnostics \\
& Vending machine control \\
\hline
\end{tabular}

Table 2.2: MTC applications

congestion, communication overload, to name a few are required to be improved. It has been reported [16] that one way to optimize the system in order to deal with these issues consists in using IPv6 addresses and grouping similar MTC devices for management purpose. In this regard, a proposed generic MTC-based architecture as defined by 3GPP [16] [15] is depicted in Fig. 2.2, which consists of three primary components, namely: MTC device domain, network domain, and MTC application domain.

- MTC device domain: this domain is composed of all MTC devices that are installed for autonomous data collection and transmission. Smoke detectors, theft control devices, fire alarms, smart meters, fitness or health monitoring devices, data collector sensors, 
tracking devices, traffic sensors, to name a few, are examples of physical MTC-based devices belonging to this domain. These devices transmit data to MTC servers or among each other.

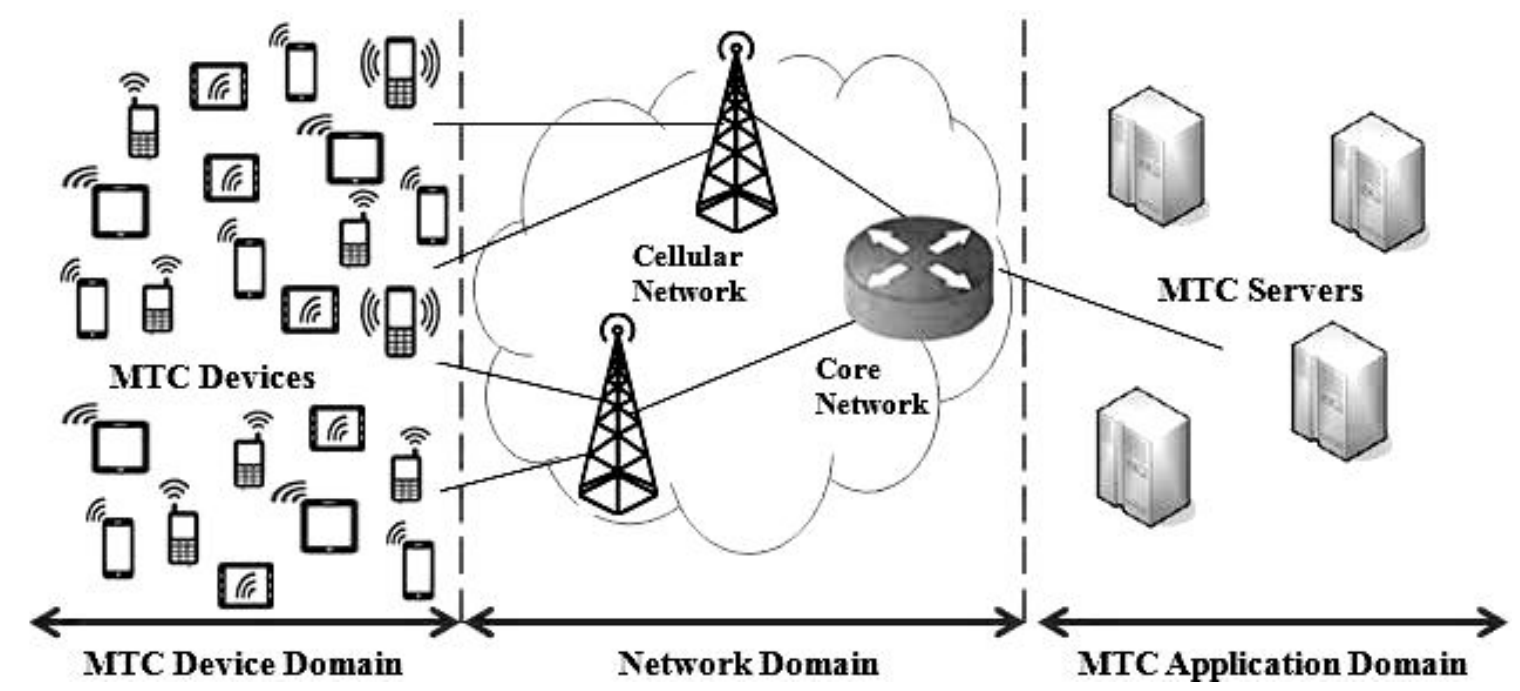

Figure 2.2: Generic architecture of MTC.

- Network domain: This domain is the backbone of the whole MTC landscape. Its goal is to provide communication between MTC devices and MTC servers or among MTC devices, through a wired or wireless network. 3GPP cellular networks such as UMTS or LTE are expected to be used as network domain for MTC applications.

- MTC application domain: This domain consists of MTC servers that serve as destination for the data transmitted by the MTC devices over the network. Based on various usage scenarios, MTC servers can be controlled and managed by mobile network operators or third party service providers [5]. MTC servers provide end users with an interface to access the assigned MTC applications.

According to 3GPP [16], the communication scenarios of MTC traffic can be segregated into two models based on various different requirements:

- Direct communication model: In this model, there is a direct communication among MTC devices which is provided by the 3GPP operator. MTC devices within the 
same network domain or different network domains can communicate to each other directly, in such a way as to establish a peer-to-peer connection [15]. Fig. 2.3 shows the communication scenario between MTC devices.

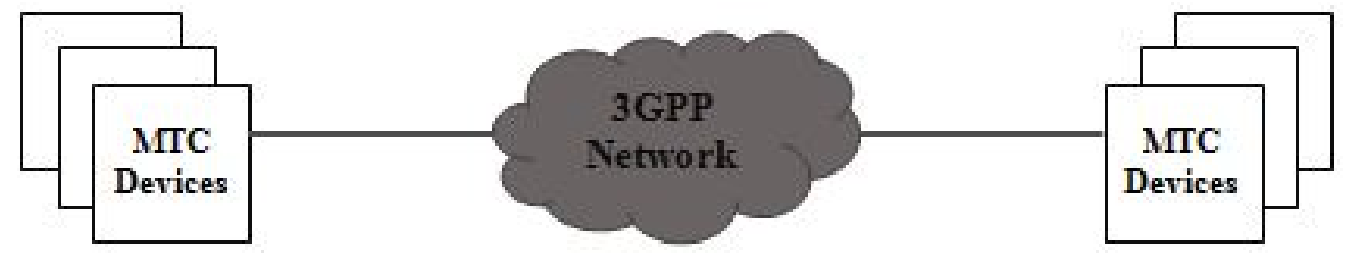

Figure 2.3: MTC Devices communicating directly with each other.

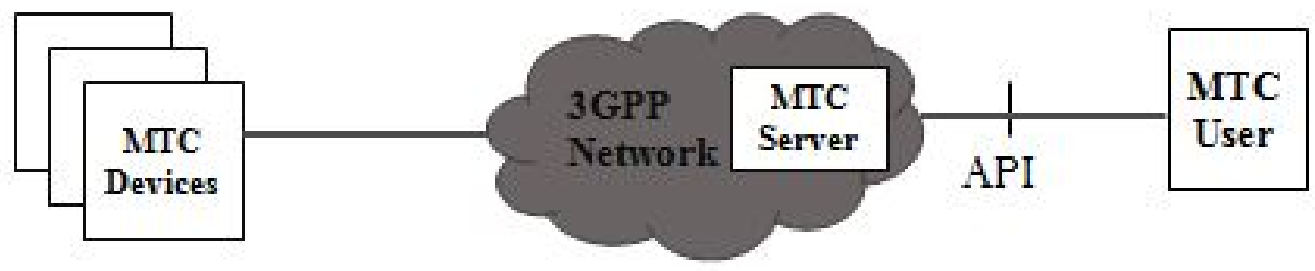

(a)

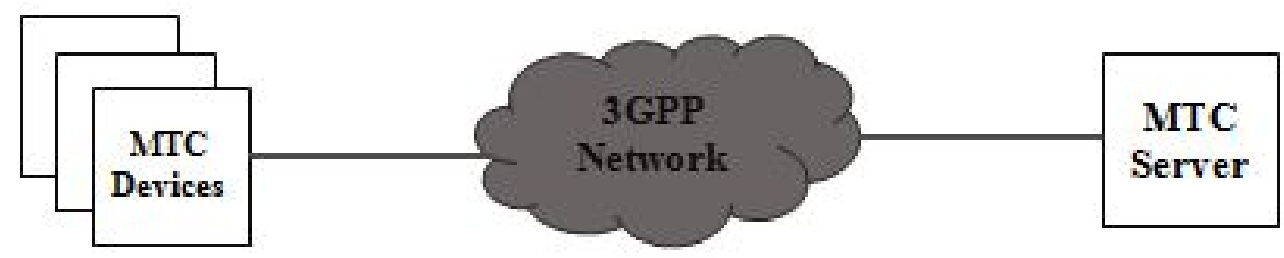

(b)

Figure 2.4: MTC Devices communicating with one or more MTC servers (a) MTC Server located inside the network domain and (b) MTC Server located outside the network domain.

- Indirect communication model: This model depicts a client-server model, where MTC 
devices (clients) transmit the data to one or more MTC servers. This scenario can find applications in smart metering, traffic controls, monitoring applications, to name a few [15]. Also, in this communication model, the MTC server can reside inside (respectively outside) the network domain, thereby, it can be controlled by the 3GPP network provider (respectively a third party service provider). When the MTC server is inside the network domain, the network provider offers an API to the MTC users for accessing the server. Fig. 2.4 shows the communication scenarios between the MTC devices and the MTC servers.

\subsubsection{Radio Resource Management}

In cellular networks, a radio resource management (RRM) scheme is required to ensure that the incoming traffic from the accepted MTC-based devices can be properly served using the available limited resources while guaranteeing that these devices will not experience a resource starvation. A RRM scheme basically aims at making the best use of limited resources and to ensure a sufficient QoS by using various strategies and resource allocation algorithms. Admission control, queue management, traffic scheduling, and power control are the most important components [17] involved in the design of an efficient RRM framework. These components can be described as follows:

- Admission control: Admission control helps the RRM scheme to ensure the QoS for both incoming and ongoing users. It helps determining the appropriate number of users to be accepted into the network. To do so, this component monitors the available radio resources and adjust the incoming and ongoing traffic accordingly. Some admission control schemes may also prioritize the users in different service classes to maintain their QoS requirements. For instance, a video call (or video streaming) would require immediate resources since any delay in data transfer will cause unsatisfactory results. On the other hand, a delay of few seconds in transmitting a pager message (data transfer) does not make much difference to the end users. Since there is no restriction 
on the movement of the mobile devices, these devices can freely move from one base station (cell) to another. Thus, prioritizing the handoff calls is an important feature of cellular networks handled by the admission control scheme.

- Queue management: Another important element of a RRM framework is queue management, which refers to how to determine the sequence of actions to be taken on the incoming packets that are in the waiting queue of the wireless transmitter prior to their transmissions. This is important since it helps avoiding some congestion in the transmission. A queue management system is meant to reduced the data transmission rates when it detects that the data packets in the queue have reached a certain level and further addition of data packets will result in the dropping of some packets.

- Scheduling: The actual transmission of the data packets out of the queue is handled by the traffic scheduling portion of the queue management scheme, which determines which queue (among the available ones) should be given priority when transmission is decided by the traffic scheduler based on the scheduling policy in place. Generally, round-robin or weighted round-robin techniques are utilized as scheduling policies.

- Power control: This component imposes some control in limiting the interference among the users of the system, so as to achieve a better transmission rate. In multi-user wireless networks such as CDMA-cellular networks, where all the users utilize the same transmission bandwidth, the transmitting power from the users can be controlled by the power control component, typically through an increased reuse of the available radio channels.

The deployment of MTC applications over a cellular network designed according to HTC traffic features creates some challenges in terms of RRM. MTC features such as small packet size, uplink transmission, small amount of traffic, low mobility of devices along with large number of devices, are quite different from HTC features. These differences contribute to the complexity of the RRM design since the allocation of radio resources to MTC traffic has 
an impact on the performance of existing HTC traffic. Therefore, designing a RRM scheme for wireless communication networks supporting both MTC traffic and HTC traffic remains

a challenge. Highly controlled power budgets and spectrum scarcity impose that the design of such RRM scheme be able to support large MTC devices while maintaining the QoS requirements for both HTC and MTC traffic. Techniques such as clustering or grouping of MTC devices [18] might help achieving such goals.

\subsection{Related Work}

In the recent years, the coexistence of MTC and HTC traffic in cellular networks has been the subject of various research investigations from a radio resource management viewpoint [6], [7], [8], [18], [19], [20], [21], [22]- [27].

Lien et al. [18] proposed a RRM method to handle MTC traffic in LTE networks, where MTC devices are grouped into clusters based on their QoS characteristics. The packet arrival rate and maximum tolerable jitter are taken into account as QoS parameters. A higher priority is given to a cluster whose packet arrival rate is larger. The radio resources are managed based on clusters instead of individual MTC devices. However, this method provides QoS guarantees only for MTC traffic and does not study the impact of MTC traffic on HTC traffic.

Bang et al. [19] proposed a user pairing-based suboptimal minimal resource allocation (SMRA) scheme with the primary objective of minimizing the amount of resource blocks to support a large number of MTC devices in LTE networks. In their proposed scheme, the resource blocks are reduced by pairing a maximum of two MTC devices that use the same resource block. The performance of SMRA is compared against the orthogonal resource allocation (ORA) scheme and the random user-pairing resource allocation (RUPRA) scheme. However, the case of regular LTE network traffic has not been considered.

Liu et al. [6] investigated the importance of categorizing the QoS of MTC and HTC 
traffic in cellular networks. A QoS categorization scheme for MTC-based services in cellular networks is proposed, which is made of eight classes, based on QoS parameters such as realtime, accuracy, and priority. A HTC QoS class category is also introduced, along with a QoS category oriented to MTC services only. However, no implementation is provided to assess and validate these schemes.

Aijaz and Aghvami [20] proposed an energy-aware RRM scheme for MTC/HTC coexistence scenarios in LTE networks, with guaranteed QoS requirements for different users. Two low complexity heuristic algorithms are proposed with the goal of minimizing the overall transmit power while assuring the QoS requirement of both HTC and MTC users. The first heuristic is shown to effectively achieve the goal of transmitting HTC and MTC data at the minimum power whereas the second one is shown to minimize the transmission power only for HTC traffic.

Various radio resource allocation schemes are presented in [21] for MTC/HTC co-existence scenarios in LTE-Advanced cellular networks, with the goal of maximizing the aggregate network utility and minimizing the co-channel interference that may be caused by the coexistence of MTC and HTC traffic. HTC and MTC services are categorized into four different classes. In the proposed RRM schemes, data rate is considered as QoS parameter.

Makris et al. [7] have focused on resource management for combined HTC and MTC traffic to provide desired QoS to both traffic types, by proposing a context-aware backhaul management (CABM) scheme for MTC gateways that provides QoS provisioning to the combined information flows originated from both HTC and MTC traffic considering various classes of HTC/MTC traffic, namely: conventional, streaming, interactive, background, priority alarm, and time tolerant or time controlled. Their RRM scheme is meant to distribute the backhaul capacity to these classes based on their nature of service and the predefined QoS. Their scheme is shown to satisfy novel MTC services without degrading the QoS for existing HTC services.

Wei et al. [8] investigated the problem of radio access networks (RANs) overload that 
result from a mass access to the network by MTC devices, which may degrade the service quality of HTC. An analytical model is proposed based on a recursive contending users estimation (RCE) technique, which helps analyzing the service quality of HTC services under the heavy load experienced by MTC traffic. The proposed analytical model is used to determine the optimal group size and required radio resource on each traffic type based on a given target access success probability.

Potsch et al. [22] studied how MTC can influence the LTE network. They have proposed a traffic model for the implementation of an MTC protocol in transport logistics. A possible logistic scenario of future MTC devices with regular HTC traffic in LTE networks is also investigated. Three types of HTC traffic (voice, video and file transfer) along with one type of MTC traffic type, are considered to investigate the influence of MTC traffic in LTE networks running HTC traffic. In their study, priority is given to voice and video services due to their delay sensitive feature. It is shown that an increasing MTC traffic load has no impact on priority services, but performance degradation is observed for file transfer services.

Giluka et al. [23] proposed a class-based priority scheduling algorithm for device to base station communication in LTE networks. In their scheme, the traffic QoS are considered as criteria to schedule the radio resources into different priority classes, i.e. from high priority class (HTC) to low priority class (MTC) applications. A threshold value is set on the MTC traffic to guarantee the QoS of HTC traffic. The performance of HTC traffic in terms of its throughput is analyzed by varying the MTC traffic.

Hsu et al. [24] proposed an enhanced cooperative access class barring (ECACB) scheme, along with a traffic adaptive radio resource management (TARRM) scheme for MTC traffic in LTE-A networks. Their proposed schemes are meant to minimize the random access delay and maximize the network throughput respectively. For the ECACB scheme, the number of MTC devices that are associated with the BS is used as a criterion to determine the access probability, which itself determines whether further MTC devices will be granted access to the BS or not. Based on the data transfer size and the random access rate, the TARRM 
scheme is responsible for allocating the radio resources to those MTC devices that have been granted access to the BS. In order to improve the network throughput, the TARRM scheme also allocates the unused resource blocks of the UEs to the MTC devices.

Lin et al. [25] proposed a prioritized random access scheme with dynamic access barring framework to solve the RAN overload problem caused by heavy load of MTC traffic in LTEA networks. The proposed scheme considers five different classes of HTC and MTC traffic, namely emergency, HTC, high priority, low priority and scheduled. Different random access channels resources are pre- allocated to different classes. A dynamic access barring scheme is used to avoid collisions due to simultaneous random access attempts by a large number of MTC traffic. The performance of the proposed scheme is evaluated by simulations, showing promising results in terms of access probability.

Lee et al. [26] have investigated the random access overload problem due to large number of MTC devices in LTE-A networks. They have analyzed the throughput performance of two candidate methods for random access preamble allocation and management, proposed for possible adoption in LTE-A networks. The first method was meant to completely split the set of available random access preambles into a subset for human-to-human $(\mathrm{H} 2 \mathrm{H})$ customers and another subset for M2M customers/devices. The second method was meant to split the set of available random access preambles into a subset for $\mathrm{H} 2 \mathrm{H}$ customers only and a subset for both $\mathrm{H} 2 \mathrm{H}$ and M2M customers. Their study showed that there is a boundary of random access load below which the second method outperforms the first method slightly, but above which the second method degrades the throughput significantly.

Ide et al. [27] proposed a channel aware MTC-based resource allocation scheme for the coexistence of HTC and MTC traffic in LTE networks, with the goal to minimize the impact of MTC traffic on HTC traffic. The key idea of their scheme is to transmit the MTC data with high probability when the channel conditions are deemed appropriate to do so. A Markovian model is used to evaluate the performance of the proposed scheme, and the impact of MTC on HTC traffic is analyzed in terms of blocking probabilities. 
In this thesis, a novel CTMC model-based RRM scheme is proposed, which allocates the radio channels to HTC traffic and MTC traffic, by introducing a dedicated shared area that provides QoS isolation between HTC traffic and MTC traffic. The effectiveness of the proposed scheme is validated by simulations in Chapter 4 . 


\section{Chapter 3}

\section{Proposed Radio Resource}

\section{Management Scheme}

This chapter covers the main contributions of our thesis since it describes our proposed CTMC-based RRM scheme used to analyze the impact of MTC traffic on HTC traffic in wireless communication networks, with respect to predefined performance metrics.

\subsection{System Model and Traffic Assumptions}

The system model consists of a BS that supports the mobile users and MTC devices using a wireless technology. In this model, $N_{c h}$ denotes the number of radio channels in the BS; two types of service classes are supported by the network, namely MTC traffic and HTC traffic, with different characteristics. For instance, MTC traffic is featured by having smaller packets size coming from numerous devices whereas a HTC has larger packets size but the number of devices are comparatively less. Only the data services in HTC traffic are assumed. The arrival processes of HTC traffic and MTC traffic follow two independent Poisson processes with parameters $\lambda_{H T C}$ and $\lambda_{M T C}$ respectively. The service time of the HTC traffic (respectively the MTC traffic) is exponentially distributed with rate $\mu_{H T C}$ (respectively $\mu_{M T C}$ ).

In order to provide a QoS isolation between MTC and HTC traffic, the proposed RRM 
scheme defines two thresholds, $K_{m}$ (for MTC traffic) and $K_{h}$ (for HTC traffic), distributed over the radio channels. Each of these thresholds is meant to ensure that a portion of the radio resources (as shown in Fig. 3.1) is dedicated to each service class. This way, $K_{m}$ radio channels are dedicated to cope with MTC traffic while $K_{h}$ are reserved for handling the HTC traffic. To increase the resource allocation flexibility, a shared area with $N_{s}=$ $N_{c h}-\left(K_{h}+K_{m}\right)$ radio channels (as shown in Fig. 3.1) is also introduced by the RRM scheme to allow the incoming requests originated from both services classes to be accepted even when there is no free resource in their dedicated areas. The flowchart illustrating the proposed RRM scheme process is depicted in Fig. 3.2.

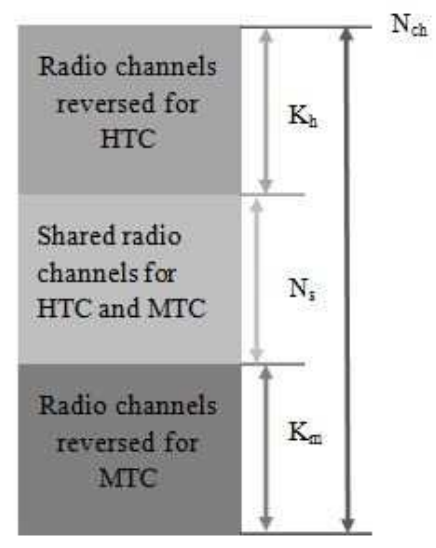

Figure 3.1: RRM scheme for HTC and MTC traffic.

The working of the flowchart in Fig. 3.2 is as follows. Whenever a service request arrives in the system, the corresponding threshold is checked against the already presented services of its class; for instance, the number of already presented HTC services $\left(S_{h}\right)$ are compared against $K_{h}$ and the MTC services $\left(S_{m}\right)$ are compared against $K_{m}$. As soon as a request for service has arrived, dedicated radio resources are checked for availability. If the threshold is not reached i.e. the dedicated resources are available, the incoming service request is accepted in the specific dedicated area for processing. In case the threshold value for the specified class has been reached by the time the service request has arrived, it is sent to be 


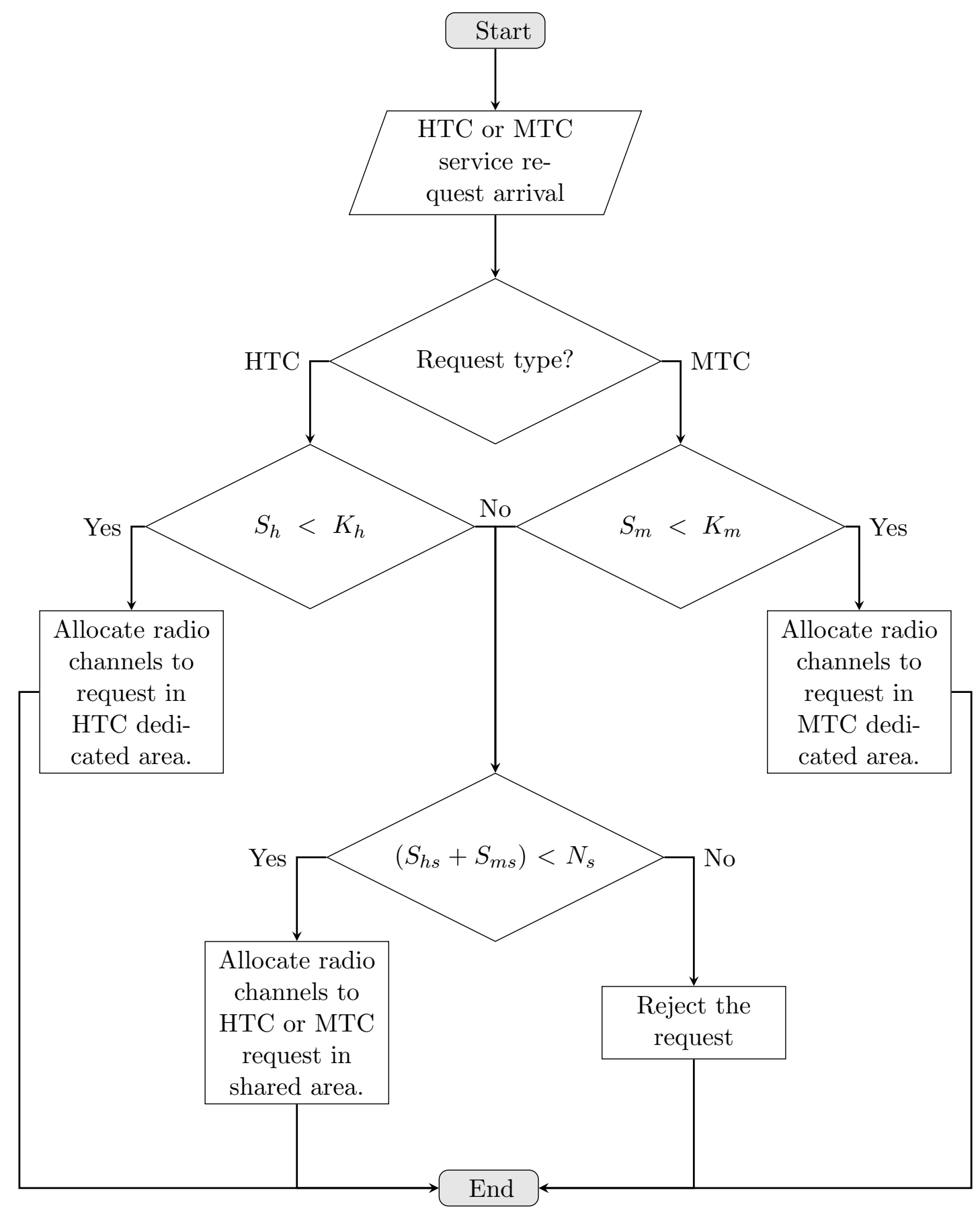

Figure 3.2: Flowchart of the proposed RRM scheme for HTC and MTC traffic.

checked against the shared area. For this the size of shared area $\left(N_{s}\right)$ is compared against the number of already presented HTC services $\left(S_{h s}\right)$ and MTC services $\left(S_{m s}\right)$ in shared area. The radio resources (i.e. $N_{s}$ radio channels) in the shared area are assigned to service the 
request based on their availability. The service request is dropped if the threshold $\left(K_{m}\right.$ or $\left.K_{h}\right)$ is reached for the specified class and the radio channels in the shared area $\left(N_{s}\right)$ are already occupied.

\subsection{CTMC-Based RRM Formulation}

A continuous-time Markov chain (CTMC) model is designed to formulate the RRM problem.

\subsubsection{States of the CTMC Model}

The states of the CTMC model are given by

$S=\left\{\left(s_{h}, s_{h s}, s_{m}, s_{m s}\right) / 0 \leq s_{h} \leq K_{h} ; 0 \leq s_{h s} \leq N_{s}-s_{m s}, 0 \leq s_{m} \leq K_{m}, 0 \leq s_{m s} \leq N_{s}-s_{h s}\right\}$

where $s_{h}$ denotes the number of HTC data packets in the HTC dedicated area; $s_{h s}$ denotes the number of HTC data packets in the shared area $N_{s}=N_{c h}-\left(K_{h}+K_{m}\right), s_{m}$ denotes the number of MTC data packets in the MTC dedicated area, and $s_{m s}$ represents the number of MTC data packets in the shared area $N_{s}$.

\subsubsection{States Transition}

The state transitions for the proposed CTMC model with their rates and conditions under which they are triggered, are shown in Table 3.1. As shown, when the number of HTC data packets in the HTC dedicated area is less than the threshold value $K_{h}$, the available radio channels will be allocated to the incoming HTC data packet. Similarly, the radio channels will be assigned to the incoming MTC data packet whenever the number of ongoing MTC data packets in the MTC dedicated area is less than the threshold value $K_{m}$. If the numbers of ongoing HTC (respectively MTC) data packets in the HTC (respectively MTC) dedicated

area is greater or equal to the threshold $K_{h}$ (respectively $K_{m}$ ), the system will redirect 
Table 3.1: State transitions

\begin{tabular}{c|c|c|l}
\hline Successor State & Condition & Rate & Event \\
\hline$\left(s_{h}+1, s_{h s}, s_{m}, s_{m s}\right)$ & $s_{h}<K_{h}$ & $\lambda_{H T C}$ & Arrival of HTC data packet in HTC dedicated area \\
\hline$\left(s_{h}, s_{h s}+1, s_{m}, s_{m s}\right)$ & $\left(s_{h}=K_{h}\right) \wedge\left(s_{h s}+s_{m s}\right)<N_{s}$ & $\lambda_{H T C}$ & Arrival of HTC data packet in the shared area \\
\hline$\left(s_{h}, s_{h s}, s_{m}+1, s_{m s}\right)$ & $s_{m}<K_{m}$ & $\lambda_{M T C}$ & Arrival of MTC data packet in MTC dedicated area \\
\hline$\left(s_{h}, s_{h s}, s_{m}, s_{m s}+1\right)$ & $\left(s_{m}=K_{m}\right) \wedge\left(s_{h s}+s_{m s}\right)<N_{s}$ & $\lambda_{M T C}$ & Arrival of MTC data packet in the shared area \\
\hline$\left(s_{h}-1, s_{h s}, s_{m}, s_{m s}\right)$ & $s_{h}>0$ & $s_{h} \mu_{H T C}$ & Departure of HTC data packet from HTC dedicated area \\
\hline$\left(s_{h}, s_{h s}-1, s_{m}, s_{m s}\right)$ & $s_{h s}>0$ & $s_{h s} \mu_{H T C}$ & Departure of HTC data packet from shared area \\
\hline$\left(s_{h}, s_{h s}, s_{m}-1, s_{m s}\right)$ & $s_{m}>0$ & $s_{m} \mu_{M T C}$ & Departure of MTC data packet from MTC dedicated area \\
\hline$\left(s_{h}, s_{h s}, s_{m}, s_{m s}-1\right)$ & $s_{m s}>0$ & $s_{m s} \mu_{M T C}$ & Departure of MTC data packet from shared area \\
\hline
\end{tabular}

further data packets to the shared area $N_{s}$ as long as the condition $\left(s_{h s}+s_{m s}\right)<N_{s}$ holds. Otherwise, an incoming data packet will be rejected. Once a HTC data packet finishes its service in the dedicated area (respectively shared area), it will leave the system with rate $s_{h} \mu_{H T C}$ (respectively $\left.s_{h s} \mu_{H T C}\right)$. A similar reasoning applies in the case of the MTC departure process.

It is impractical to graphically represent a complete state transition diagram of the proposed CTMC model due to its complexity. Therefore, to illustrate the state transitions of CTMC model we consider a particular state $\mathrm{s}=\left(S_{h}=1, S_{h s}=1, S_{m}=1, S_{m s}=1\right) \in \mathrm{S}$ and the system has six radio channels to serve HTC and MTC services. Radio channels are distributed as follows: two radio channels are dedicated to HTC, two radio channels are dedicated to MTC, and two radio channels are allocated to the shared area. The state diagram of all possible transitions to state s are shown in Fig. 3.3(a) and transitions from state s are shown in Fig. 3.3(b). As shown in the Fig. 3.3, the system moves to state s or from state s upon an arrival or a departure of a HTC (MTC) services with rate $\lambda_{H T C}\left(\lambda_{M T C}\right)$ or $\mu_{H T C}\left(\mu_{M T C}\right)$ respectively. For instance, in Fig. 3.3(a) the system will move to state $\mathrm{s}=$ $(1,1,1,1)$ from state $s^{\prime}=(0,1,1,1)$ with arrival rate $\lambda_{H T C}$ or from state $s^{\prime}=(2,1,1,1)$ with departure rate $S_{h} \mu_{H T C}$. Similarly in Fig. 3.3(b) the system will move from state s $=(1,1,1,1)$ to state s' $=(1,1,0,1)$ with departure rate $S_{m} \mu_{M T C}$ or from state s' $=(1,1,2,1)$ with arrival rate $\lambda_{M T C}$. 


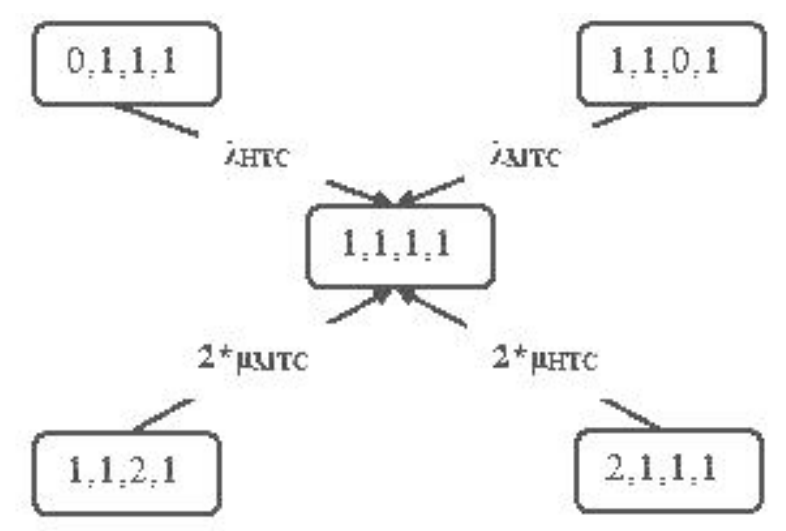

(a)

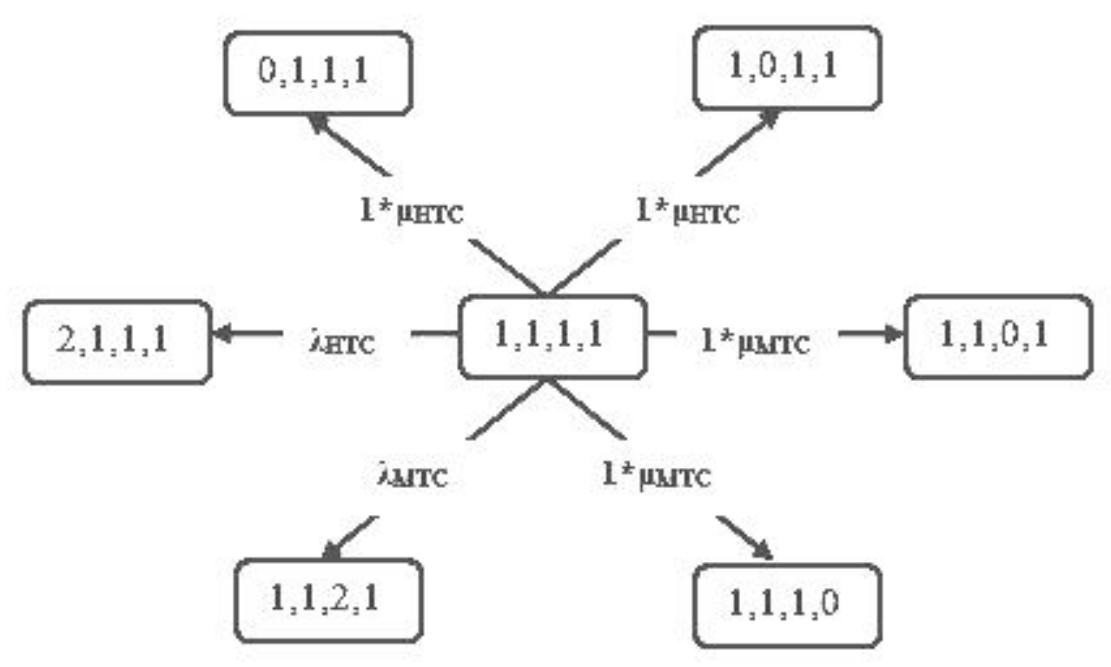

(b)

Figure 3.3: State transition diagram of state s of the CTMC model (a) Transitions to state $s=(1,1,1,1) \in \mathrm{S}$ and (b) Transitions from state $s=(1,1,1,1) \in \mathrm{S}$.

\subsubsection{Blocking Probabilities and Channel Utilization}

Let $\pi\left(s_{h}, s_{h s}, s_{m}, s_{m s}\right)$ denote the CTMC model steady-state probability. The blocking probability $\left(P b_{H T C}\right)$ of the HTC data packets is defined as the probability that the number of HTC data packets in the HTC dedicated area is equal to the threshold $K_{h}$ and the number 
of HTC and MTC data packets in the shared area is equal to the size of shared area $N_{s}$, i.e.

$$
P b_{H T C}=\sum_{s_{h}=K_{h}} \sum_{\left(s_{h s}+s_{m s}\right)=N_{s}} \pi\left(s_{h}, s_{h s}, s_{m}, s_{m s}\right)
$$

Similarly, the blocking probability $\left(P b_{M T C}\right)$ of the MTC data packets is defined as the probability that the number of MTC data packets in the MTC dedicated area is equal to the threshold $K_{m}$ and the number of HTC and MTC data packets in the shared area is equal to the size of shared area $N_{s}$, i.e.

$$
P b_{M T C}=\sum_{s_{m}=K_{m}} \sum_{\left(s_{h s}+s_{m s}\right)=N_{s}} \pi\left(s_{h}, s_{h s}, s_{m}, s_{m s}\right)
$$

Furthermore, the channel utilization $C h_{H T C}$ for the HTC traffic is defined as the ratio between the mean number of busy channels serving the HTC data packets and the total number of radio channels, i.e.

$$
C h_{H T C}=\frac{\sum_{s_{h}>0, s_{h s}>0, s_{m} \geq 0, s_{m s} \geq 0}\left(s_{h}+s_{h s}\right) \pi\left(s_{h}, s_{h s}, s_{m}, s_{m s}\right)}{N_{c h}}
$$

Similarly, the channel utilization $C h_{M T C}$ for the MTC traffic is defined as the ratio between the mean number of busy channels serving the MTC data packets and the total number of radio channels., i.e.

$$
C h_{M T C}=\frac{\sum_{s_{h} \geq 0, s_{h s} \geq 0, s_{m}>0, s_{m s}>0}\left(s_{m}+s_{m s}\right) \pi\left(s_{h}, s_{h s}, s_{m}, s_{m s}\right)}{N_{c h}}
$$

Finally, the channel utilization $C h_{\text {Shared }}$ of the shared area is the ratio between the mean number of busy channels serving either HTC or MTC data packets in the shared area and 
the total number of radio channels, i.e.

$$
C h_{\text {Shared }}=\frac{\sum_{s_{h} \geq 0, s_{h s}>0, s_{m} \geq 0, s_{m s}>0}\left(s_{m s}+s_{h s}\right) \pi\left(s_{h}, s_{h s}, s_{m}, s_{m s}\right)}{N_{c h}}
$$

The above parameters will be used as performance metrics (in Chapter 4) to assess the effectiveness of the proposed CTMC-based RRM model. 


\section{Chapter 4}

\section{Performance Evaluation}

This Chapter studies the performance of the proposed CTMC-based RRM scheme. In our performance studies, the blocking probability and channel utilization of HTC, MTC and shared areas are studied against the arrival rate of the MTC traffic, using an analytical model.

\subsection{Network Parameters}

Table 4.1 outlines the network parameters used in the numerical evaluation.

\begin{tabular}{||c||c||}
\hline \hline Network Parameters & Value \\
\hline \hline Total number of radio channels $\left(N_{c h}\right)$ & 30 \\
\hline Arrival rate of HTC traffic & 1 data packet per second \\
\hline Arrival rate of MTC traffic & varies from 1 to 10 data packets per second \\
\hline HTC data packets & are larger in size than MTC data packets \\
\hline Service time for HTC & $2 \mathrm{~s} /$ packet \\
\hline Service time for MTC & $1 \mathrm{~s} /$ packet \\
\hline
\end{tabular}

Table 4.1: Network parameters. 


\subsection{Scenarios Under Analysis}

The following scenarios are considered:

- Scenario I: The arrival rate of the MTC traffic is varied and the impact of this variation on the blocking probability of HTC data packets is studied.

- Scenario II: The arrival rate of the MTC traffic is varied and the impact of this variation on the blocking probability of MTC data packets is studied.

- Scenario III: The arrival rate of the MTC traffic is varied and the impact of this variation on the channel utilization for HTC traffic is studied.

- Scenario IV: The arrival rate of the MTC traffic is varied and the impact of this variation on the channel utilization for MTC traffic is studied.

- Scenario $V$ : The arrival rate of the MTC is varied and the impact of this variation on the channel utilization for the shared area is studied.

- Scenario VI: The arrival rate of the HTC is varied and the impact of this variation on the channel utilization for the shared area is studied.

\subsection{Performance Metrics}

To analyze the impact of MTC traffic on HTC traffic, the following performance metrics are considered, using the different set of threshold which are selected based on the previous studies [28]. values given in Table 4.2:

- The blocking probability $\left(P b_{H T C}\right)$ of the HTC data packets given by Equation 3.2.

- The blocking probability $\left(P b_{M T C}\right)$ of the MTC data packets given by Equation 3.3.

- The channel utilization $C h_{H T C}$ for the HTC traffic given by Equation 3.4. 
- The channel utilization $C h_{H T C}$ for the MTC traffic given by Equation 3.5.

- The channel utilization $C h_{\text {Shared }}$ of the shared area given by Equation 3.6.

\begin{tabular}{cc|cc|cc}
\hline \hline Thresholds Set I & \multicolumn{2}{|c|}{ Thresholds Set II } & \multicolumn{2}{c}{ Thresholds Set III } \\
\hline$K_{h}$ & $K_{m}$ & $K_{h}$ & $K_{m}$ & $K_{h}$ & $K_{m}$ \\
\hline 10 & 6 & 6 & 9 & 8 & 9 \\
12 & 9 & 10 & 9 & 8 & 12 \\
14 & 12 & 14 & 9 & 8 & 15 \\
\hline
\end{tabular}

Table 4.2: Threshold values.

\subsection{Numerical Results}

This section discusses the performance of the proposed RRM scheme and analysis the impact of MTC traffic on HTC traffic using the aforementioned performance metrics and scenarios.

\subsubsection{Scenario I: Impact of the Variation of the Arrival Rate of MTC traffic on the Blocking Probability of HTC Traffic}

The arrival rate of the MTC traffic is varied and the impact of this variation on the blocking probability of HTC data packets is investigated. The results are captured in Fig. 4.1, Fig. 4.2, Fig. 4.3 and Fig. 4.4 .

In Fig 4.1, it can be observed that when the arrival rate of the MTC traffic increases, the blocking probability of the HTC traffic also increases, thereby the chances of having the HTC packets dropped is very high. This relationship between the blocking probability of HTC traffic and the MTC arrival rate is also impacted by the change in the threshold for MTC, HTC, and shared areas. Indeed, Fig 4.1 shows that if the threshold values for HTC and MTC areas are set to lower values (e.g. $K_{h}=10$ and $K_{m}=6$ ) and the threshold of the shared area is set to be greater than $K_{h}$ and $K_{m}$, the blocking probability of HTC traffic increases sharply when the arrival rate of the MTC traffic increases. Thus, keeping lower threshold values has a greater impact on the arrival of HTC traffic. Lower threshold 


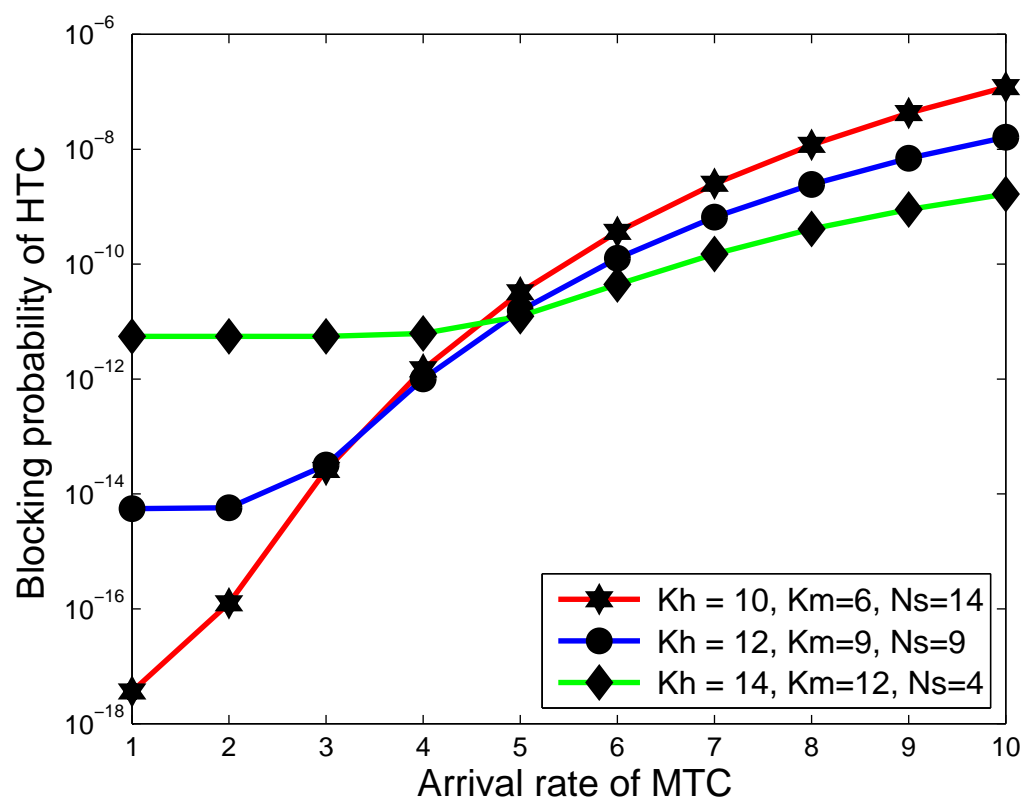

Figure 4.1: Blocking probability of HTC vs. arrival rate of MTC with varied thresholds.

values ultimately result in bigger shared area, which is filled out by the MTC traffic, leading to chances of having more HTC data packets dropped. On the other hand, the results in Fig 4.1 also show that if the thresholds are set to higher values (e.g. $K_{h}=14$ and $K_{m}=12$ ), the impact of the arrival rate of MTC traffic is not significant on the blocking probability of the HTC traffic.

Similarly, the blocking probability of the HTC traffic is analyzed by keeping the MTC threshold value constant. The results are shown in Fig 4.2. It can been noticed that if the arrival rate of the MTC traffic is low, its impact on the HTC blocking probability is negligible. This is due to the fact that all the incoming MTC requests are being served in the MTC dedicated area. However, as the arrival rate of the MTC traffic increases, the blocking probability of the HTC traffic increases sharply. This observation is caused by the usage of the shared area by the MTC traffic. Larger thresholds for HTC traffic mean that there are more dedicated resources for the HTC requests; which imply lower HTC blocking probabilities.

Now, the blocking probability of the HTC traffic is analyzed by keeping the HTC thresh- 


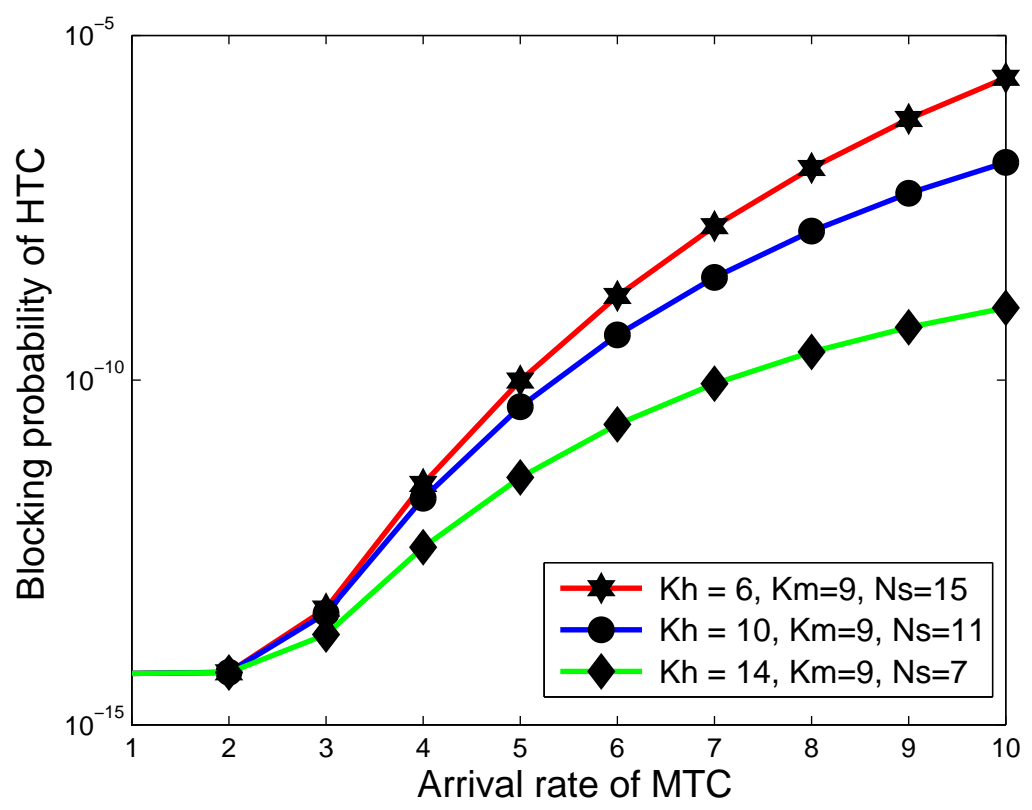

Figure 4.2: Blocking probability of HTC vs. arrival rate of MTC with constant MTC threshold.

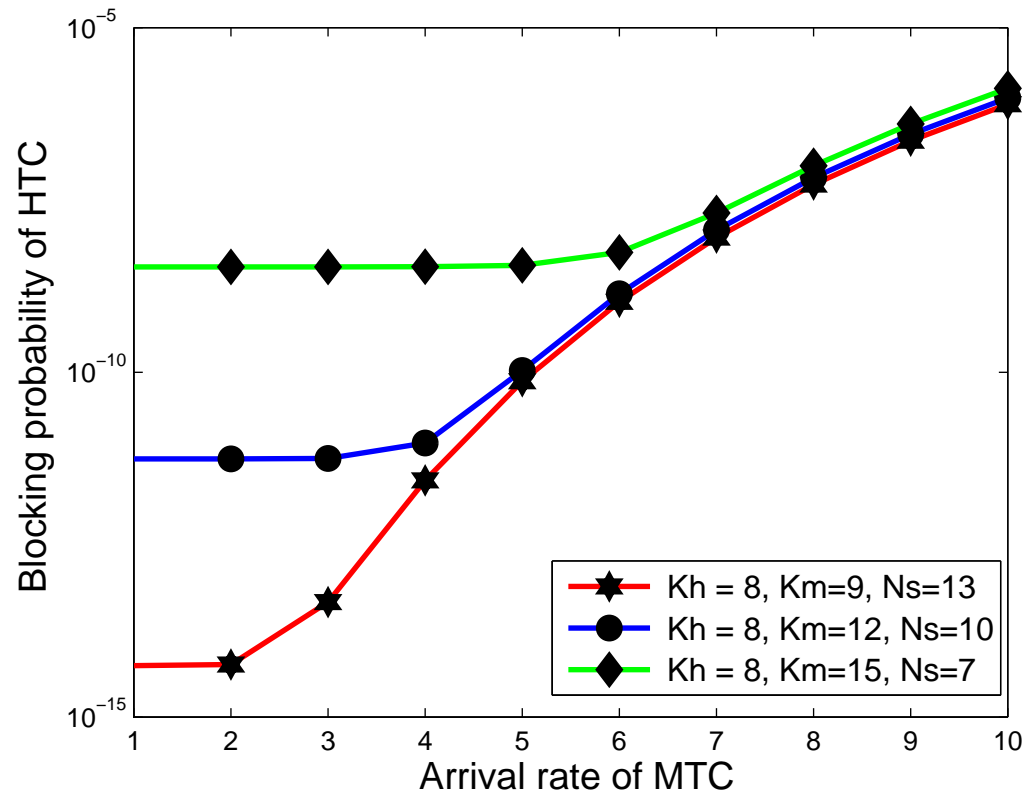

Figure 4.3: Blocking probability of HTC vs. arrival rate of MTC with constant HTC threshold.

old value constant. The results are shown in Fig 4.3. It is observed that when the arrival rate of the MTC traffic increases, the blocking probability of the HTC traffic also increases. 
But, this trend is also dependent on the threshold of the MTC traffic. For a large threshold of the MTC traffic, a slight increase is observed on the HTC blocking probability when the MTC arrival rate increases. When the MTC threshold value is small, the rate of change in the HTC blocking probability is not significant. It is also noticed that once the arrival rate of the MTC traffic reaches a particular level (here greater or equal to 7) the dependency of blocking probability of HTC on the MTC threshold value becomes negligible.

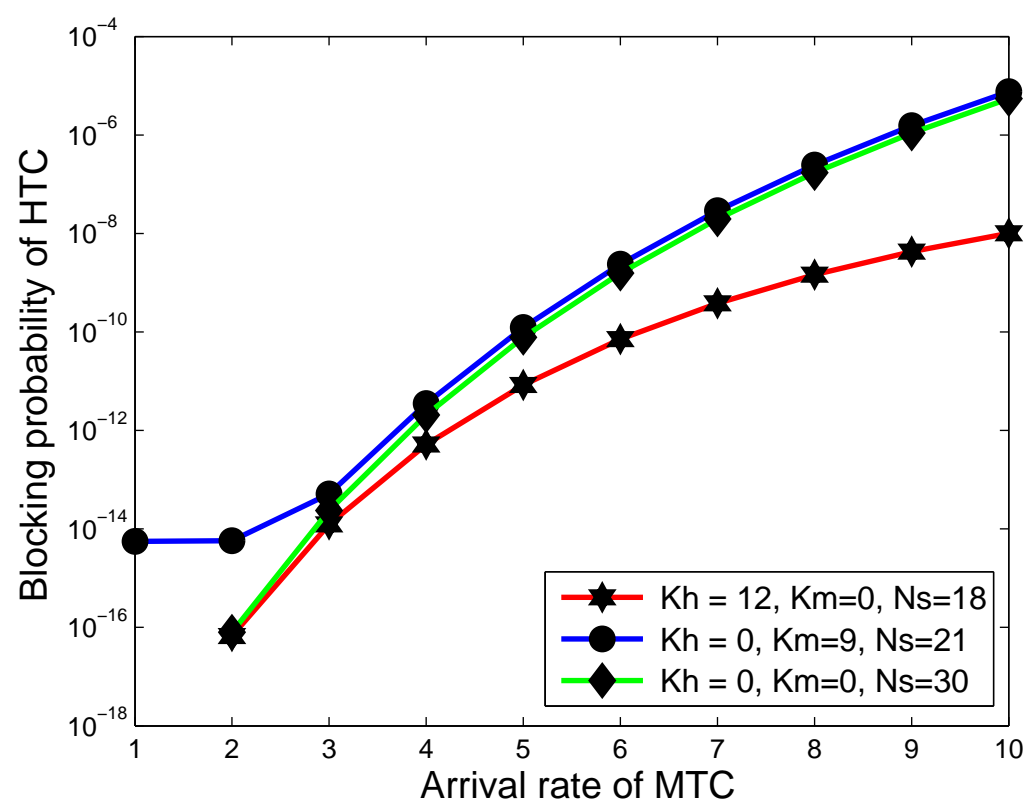

Figure 4.4: Blocking probability of HTC vs. arrival rate of MTC without thresholds.

Further, the blocking probability of HTC against arrival rate of MTC is analyzed when thresholds are not assigned to HTC and MTC traffic. Results are shown in Fig 4.4. When no thresholds are assigned to both MTC and HTC traffic (e.g. $K_{h}=0$ and $K_{m}=0$ ), the blocking probability of HTC keeps on increasing with increase in MTC arrival rate. This is because the same numbers of resources are being used for both MTC and HTC. Higher MTC arrival rate means shared area is mostly filled out with MTC requests which lead to high blocking probability. Similarly, the blocking probability of HTC is high, when no threshold is assigned to HTC. Although blocking probability is lower when some radio channels are reversed for HTC (here $K_{h}=12$ ). In this case HTC requests are served with radio channels dedicated to 
it and can also use the rest of the radio channels in shared area.

\subsubsection{Scenario II: Impact of the Variation of the Arrival Rate of MTC Traffic on the Blocking Probability of MTC Traffic}

The arrival rate of the MTC traffic is varied and the impact of this variation on the blocking probability of MTC data packets is investigated. The results are captured in Fig 4.5, Fig 4.6, Fig 4.7 and Fig 4.8 .

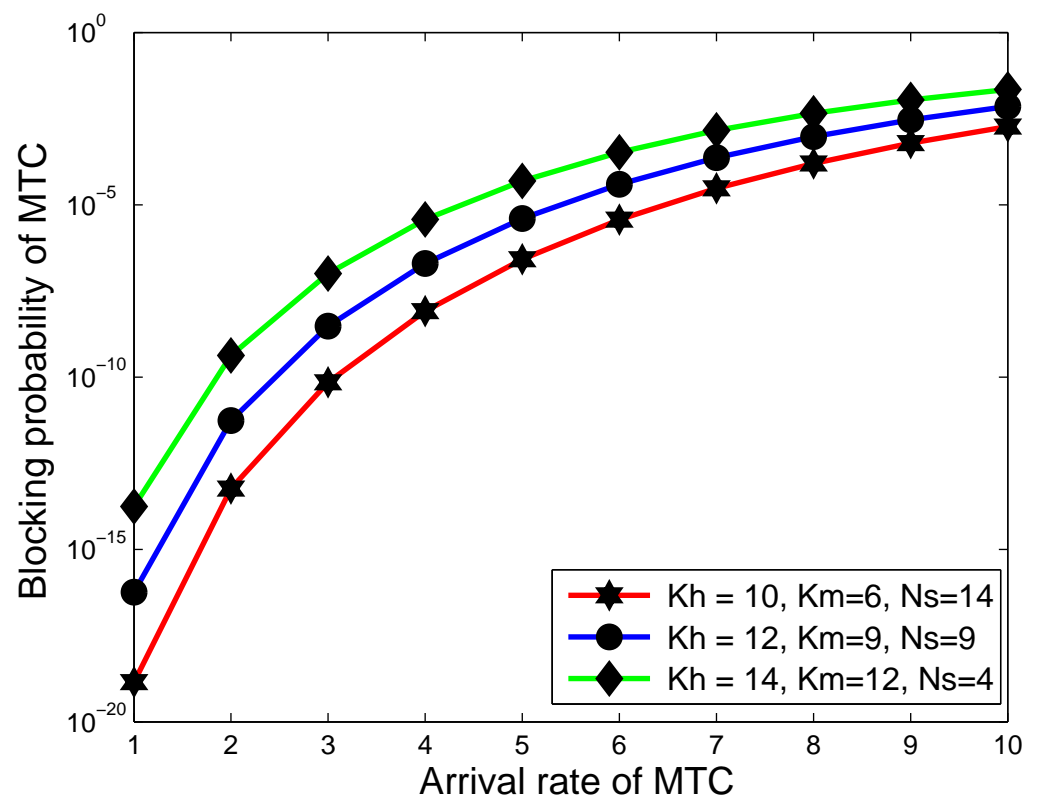

Figure 4.5: Blocking probability of MTC vs. arrival rate of MTC with varied thresholds.

In Fig. 4.5, it can be observed when the arrival rate of the MTC traffic increases, the blocking probability of the MTC traffic also increases. When the arrival rate of the MTC traffic is low, the rate at which the blocking probability of the MTC traffic increases is high; and when the arrival rate of the MTC traffic is high, the rate at which the blocking probability of the MTC traffic increases become low. In Fig. 4.5, it is also observed that if the threshold values of the MTC and HTC (i.e. $K_{m}$ and $K_{h}$ respectively) are kept at lower values, the probability of dropping the MTC data packets remain low. This is attributed to 
the fact that the shared area keeps serving the MTC requests. The HTC arrival rate was kept constant, which implies that a major portion of the shared area is used to serve the MTC traffic. Thus, lower threshold values $\left(K_{m}\right.$ and $\left.K_{h}\right)$ would create a bigger shared area, and thereby a better service for MTC traffic.

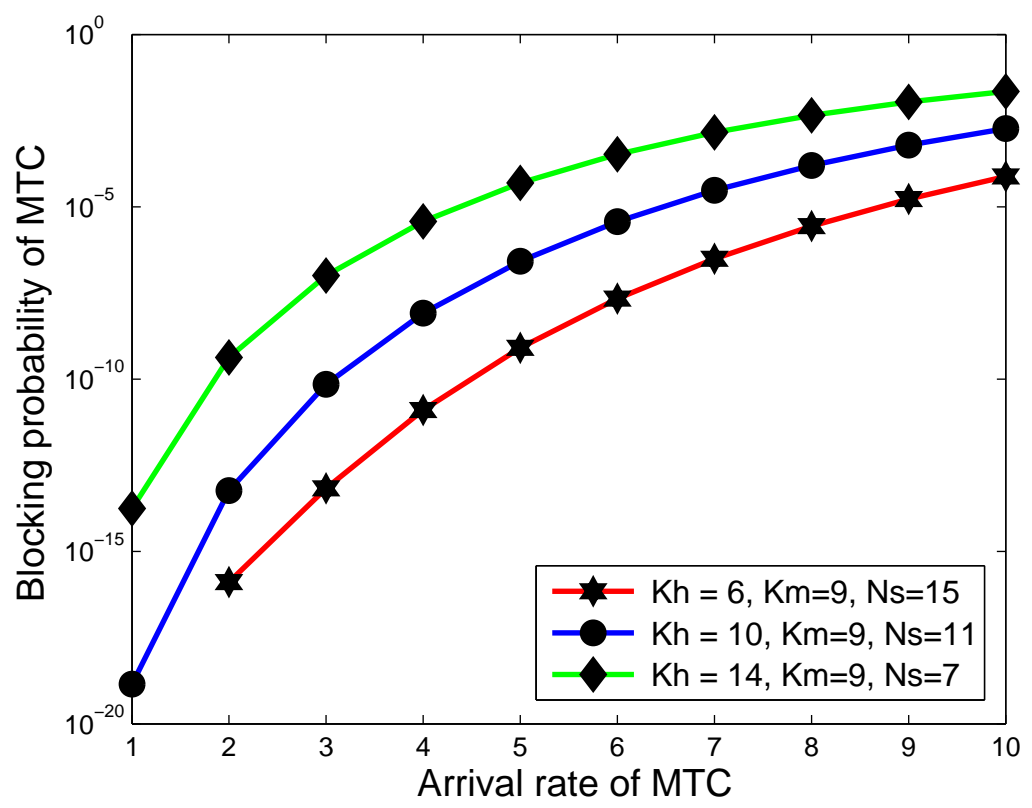

Figure 4.6: Blocking probability of MTC vs. arrival rate of MTC with constant MTC threshold.

Next, the blocking probability of the MTC traffic is analyzed when keeping the MTC threshold value constant. The results are shown in Fig. 4.6. It can be observed that when the arrival rate of the MTC traffic increases, the blocking probability of the MTC traffic greatly increases, which is attributed to the fact that more requests are to be served with the same number of available resources. Similarly, an increase in the HTC threshold reduces the shared area resources, which in turn will increase the blocking probability of the MTC traffic.

Similarly, when the HTC threshold is kept constant (here $K_{h}=8$ ), the results of the blocking probability of the MTC traffic is analyzed when keeping the HTC threshold constant. The results are shown in Fig. 4.7. It can be observed that when the arrival rate of 
MTC traffic increases, the blocking probability of the MTC traffic also increases. Similar results are obtained for each of the three MTC thresholds $\left(K_{m} 9,12,15\right)$. This is due to the availability of the same number of combined resources for the MTC and shared areas.

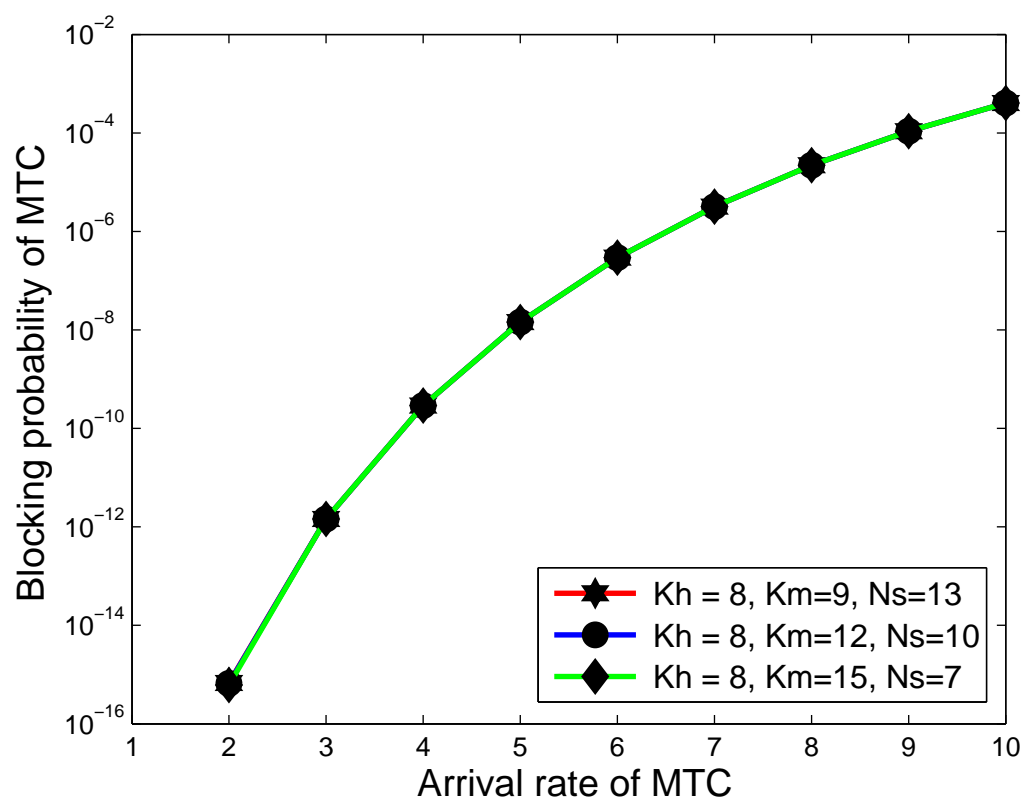

Figure 4.7: Blocking probability of MTC vs. arrival rate of MTC with constant HTC threshold.

Further, the blocking probability of MTC against arrival rate of MTC is analyzed when thresholds are not assigned to HTC and MTC traffic. Results are shown in Fig. 4.8. When no thresholds are assigned to both MTC and HTC traffic (e.g. $K_{h}=0$ and $K_{m}=0$ ), the blocking probability of MTC increases with increase in MTC arrival rate. This is due to the fact that both MTC and HTC requests are using the common radio resources of shared area. Higher MTC arrival rate means high radio resource usage and less resource availability to serve incoming requests which leads to high blocking probability of MTC. Similar results are obtained when threshold is assigned to MTC. Blocking probability of MTC is higher when some resources are reserved for HTC (e.g. $K_{h}=12$ and $K_{m}=0$ ). In this case portion of radio resources are dedicated to HTC and this reduces the size of shared area. So the number of available resources for MTC are reduced which increase the blocking probability of MTC. 


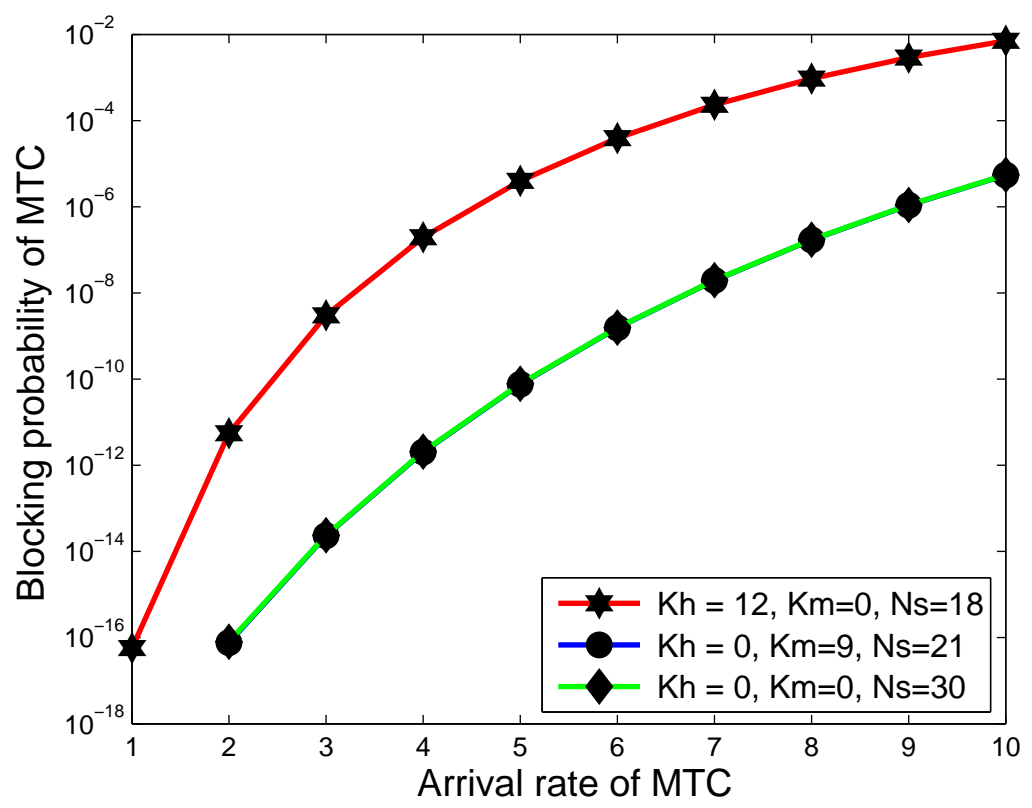

Figure 4.8: Blocking probability of MTC vs. arrival rate of MTC without thresholds.

In summary, it can be concluded that the blocking probability of the HTC traffic (respectively the MTC traffic) falls in a better range when the threshold value $K_{h}$ for the HTC traffic (respectively $K_{m}$ for the MTC traffic) is kept to about half of the number of available radio channels (respectively one third of the available radio channels). By better range, we mean that the system will likely respond to HTC and MTC traffic in a manner that less number of data packets from both type of traffic will be dropped.

\subsubsection{Scenario III: Impact of the Variation of the Arrival Rate of MTC traffic on the Channel Utilization for HTC traffic}

The arrival rate of the MTC traffic is varied and the impact of this variation on the channel utilization for HTC traffic is studied. The results are captured in Fig. 4.9, Fig. 4.10 and Fig. 4.11.

In Fig. 4.9, it can be observed that the channel utilization for HTC remains constant when the arrival rate of the MTC traffic increases. This is attributed to the fact that the HTC arrival rate was set to be constant. In this case, the variation in the threshold values 
$\left(K_{h}\right.$ and $K_{m}$ ), as well as in the arrival rate of the MTC traffic has no impact on the channel utilization of the HTC traffic.

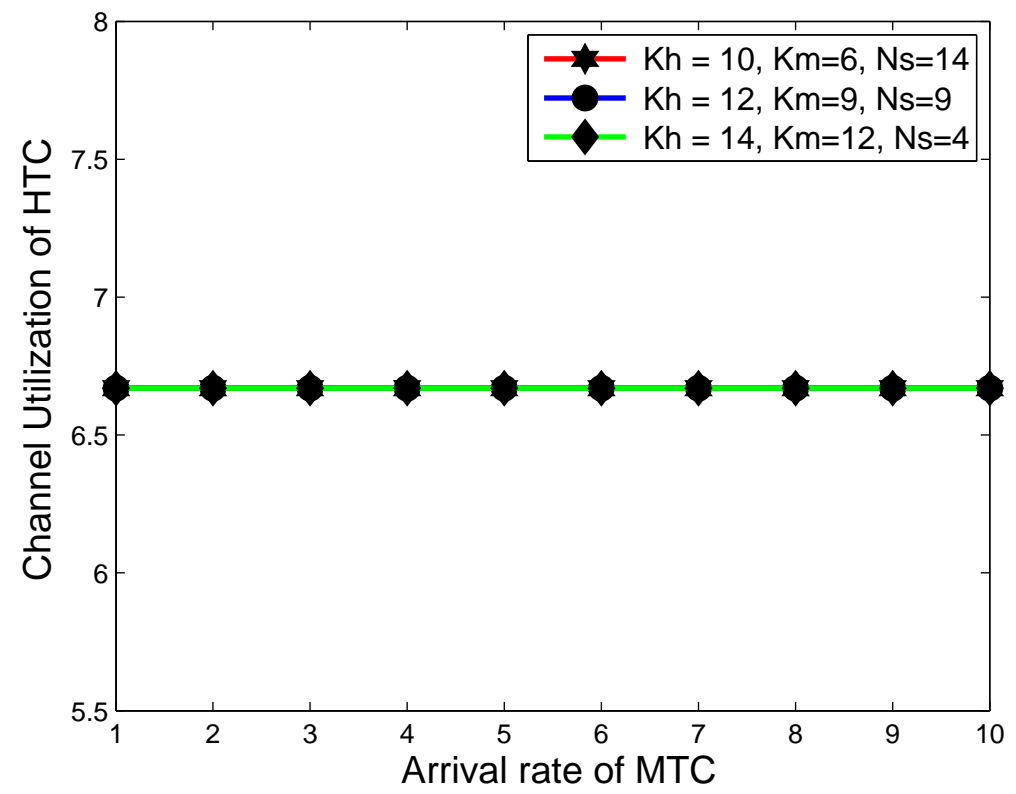

Figure 4.9: Channel Utilization for HTC vs. arrival rate of MTC with varied thresholds.

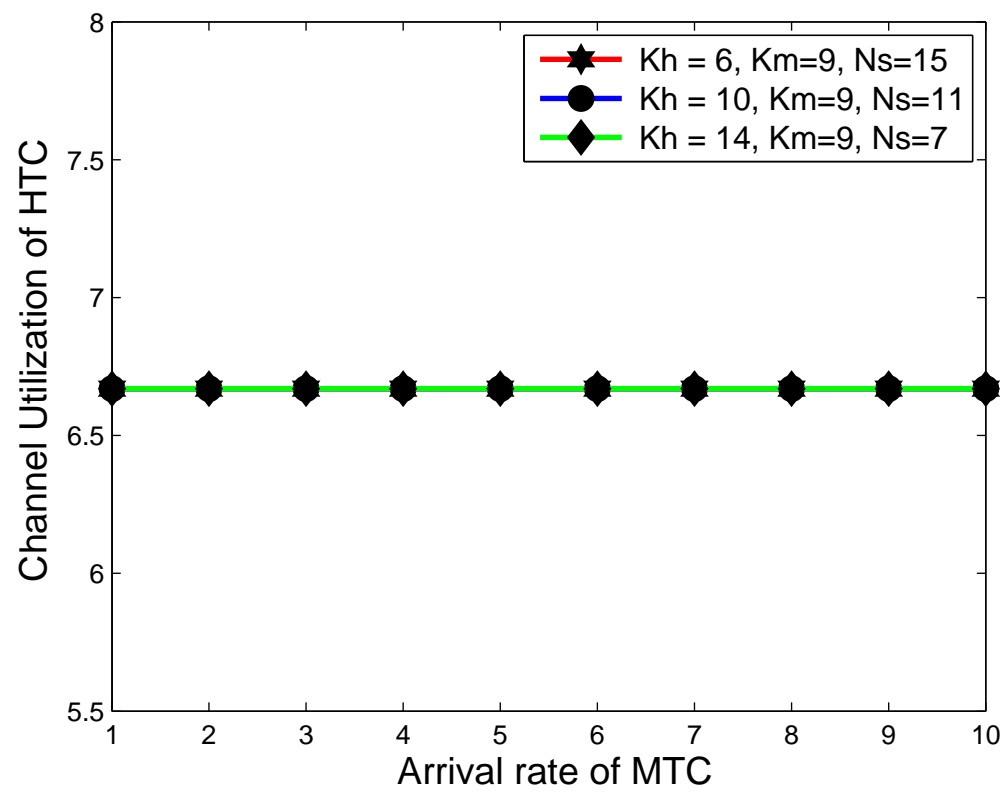

Figure 4.10: Channel Utilization for HTC vs. arrival rate of MTC with constant MTC threshold. 


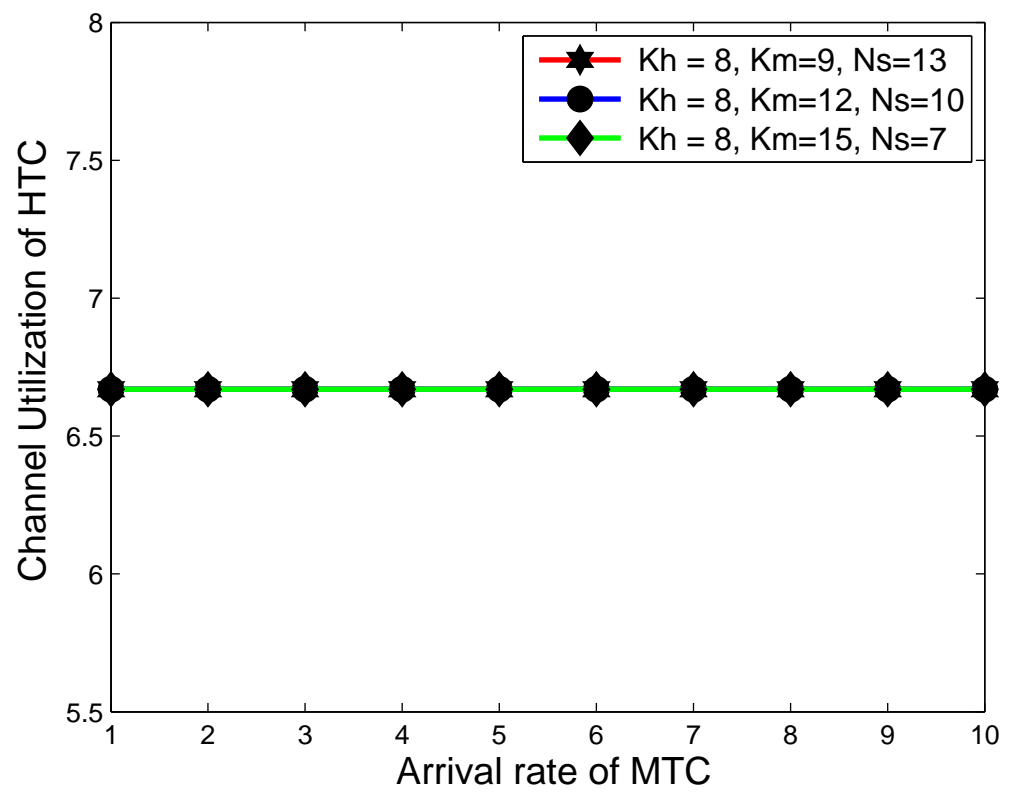

Figure 4.11: Channel Utilization for HTC vs. arrival rate of MTC with constant HTC threshold.

On the same vain, the channel utilization for HTC traffic is analyzed by keeping the MTC threshold constant (respectively the HTC threshold constant). The results are depicted in Fig. 4.10 (respectively Fig. 4.11). In both figures, the same observations as above prevail, i.e. the channel utilization for HTC remains constant when the arrival rate of the MTC traffic increases.

\subsubsection{Scenario IV: Impact of the Variation of the Arrival Rate of MTC traffic on the Channel Utilization for MTC traffic}

The arrival rate of the MTC traffic is varied and the impact of this variation on the channel utilization for MTC traffic is studied. The results are shown in Fig 4.12 Fig 4.13 and Fig 4.14.

In all three figures, it can be observed that when the arrival rate of the MTC traffic increases, the channel utilization for the MTC traffic increases linearly. In Fig 4.12 and Fig 4.13 , the variation in the threshold values $\left(K_{h}\right.$ and $\left.K_{m}\right)$ has less impact on the channel utilization of the MTC traffic. 


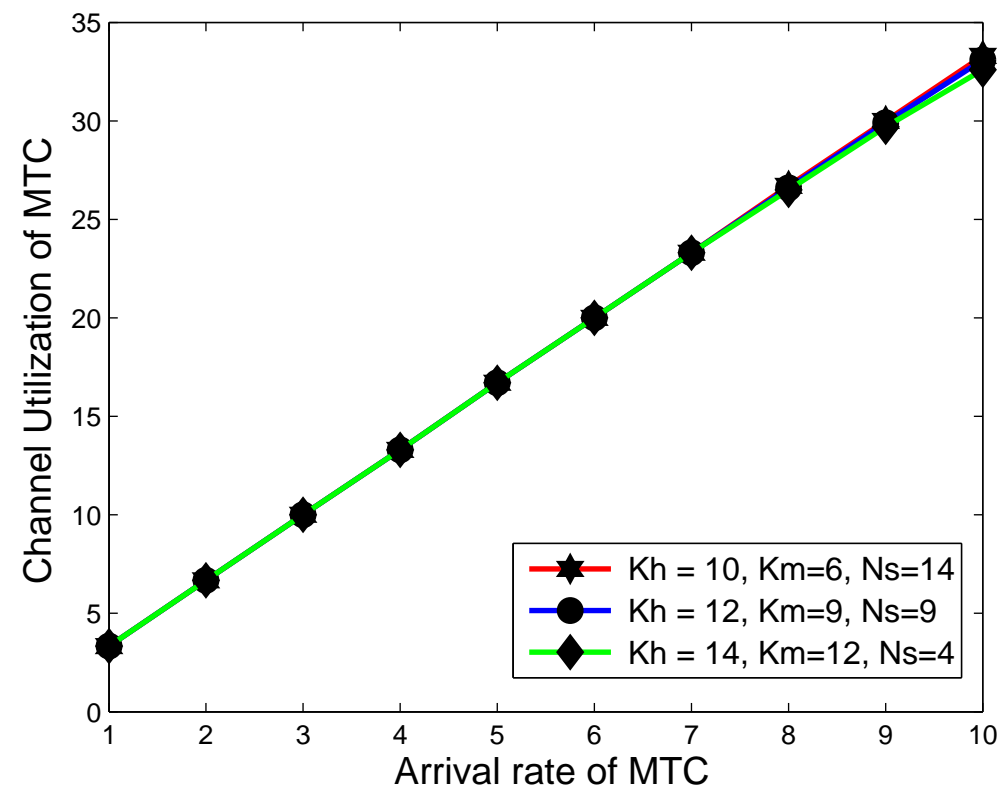

Figure 4.12: Channel Utilization for MTC vs. arrival rate of MTC with varied thresholds.

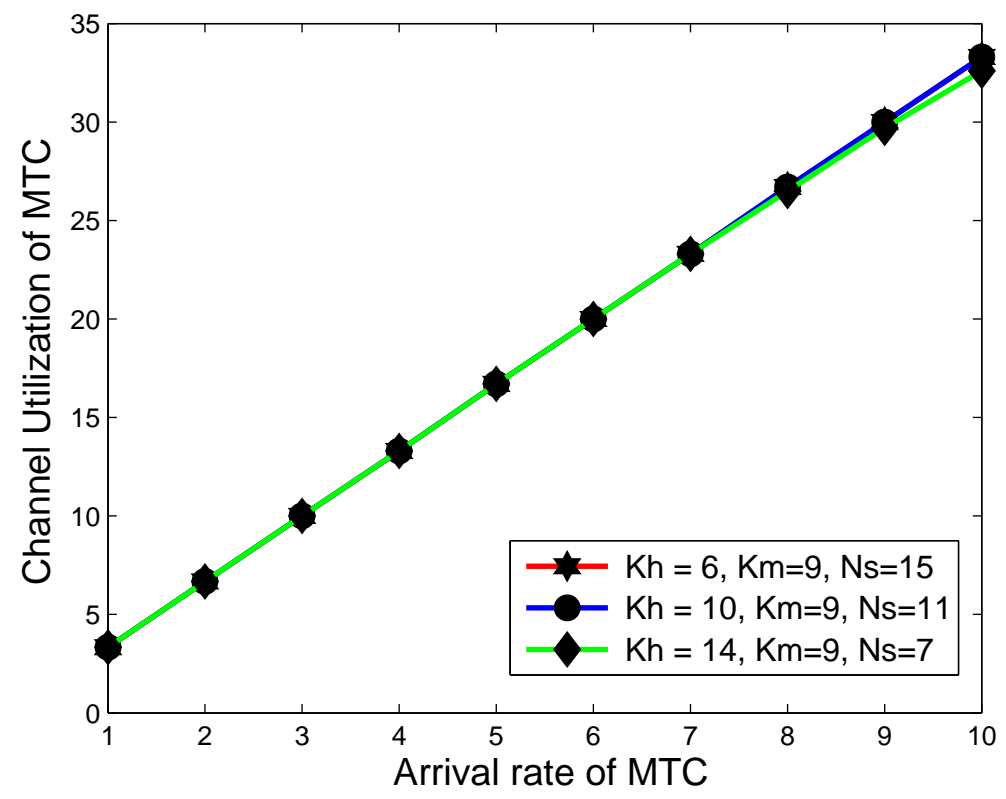

Figure 4.13: Channel Utilization for MTC vs. arrival rate of MTC with constant MTC threshold.

In Fig 4.14, it is observed that when the HTC threshold is kept constant then the channel utilization for the MTC traffic also increases linearly. These are attributed to the fact that an increase in the arrival rate of MTC traffic implies that more MTC requests are being 
handled by the system, thereby, more of the available radio channels are used.

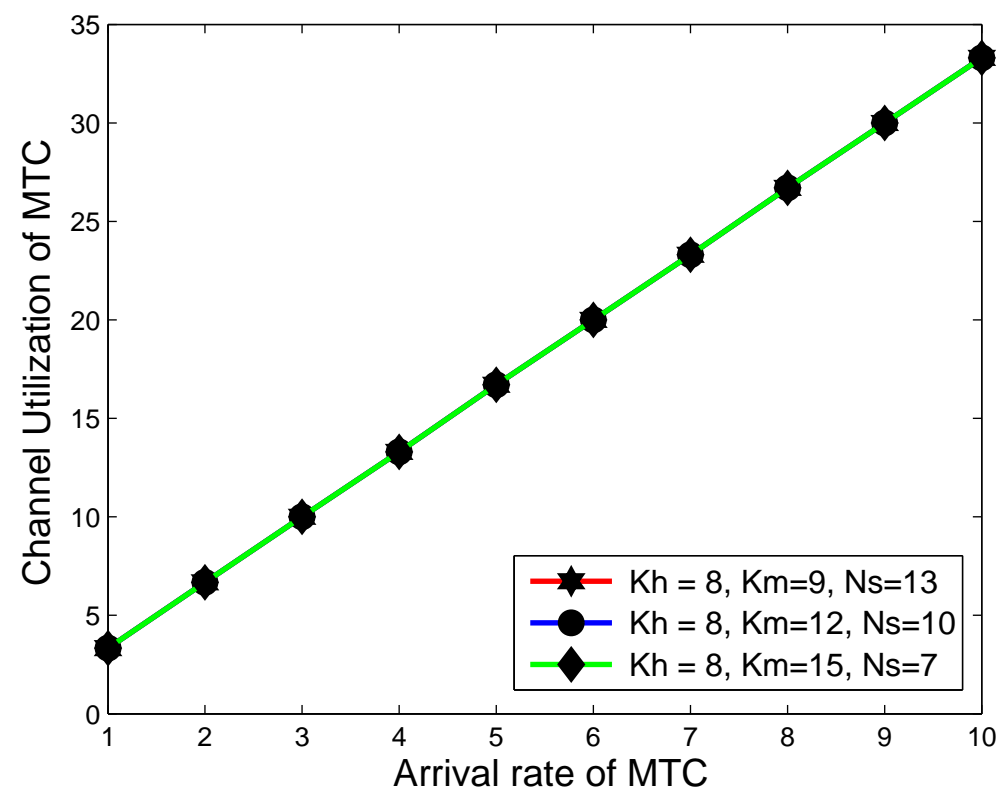

Figure 4.14: Channel Utilization for MTC vs. arrival rate of MTC with constant HTC threshold.

\subsubsection{Scenario V: Impact of the Variation of the Arrival Rate of MTC traffic on the Channel Utilization for the Shared Area}

The arrival rate of the MTC is varied and the impact of this variation on the channel utilization for the shared area is studied. The results are captured in Fig 4.15, Fig 4.16, Fig 4.17and Fig 4.18.

In Fig. 4.15, it can be observed that when the arrival rate of the MTC traffic is low, the channel utilization for the shared area remains low. This is attributed to the fact that both the HTC traffic and MTC traffic are handled by the dedicated radio channels. It can also be observed that the channel utilization of the shared area increases exponentially when the arrival rate of the MTC traffic reaches a particular level. It is also observed that when the threshold are set to lower values, a bigger shared area is obtained; thus these thresholds are reached quickly, and the utilization of the shared area is greatly increased. 


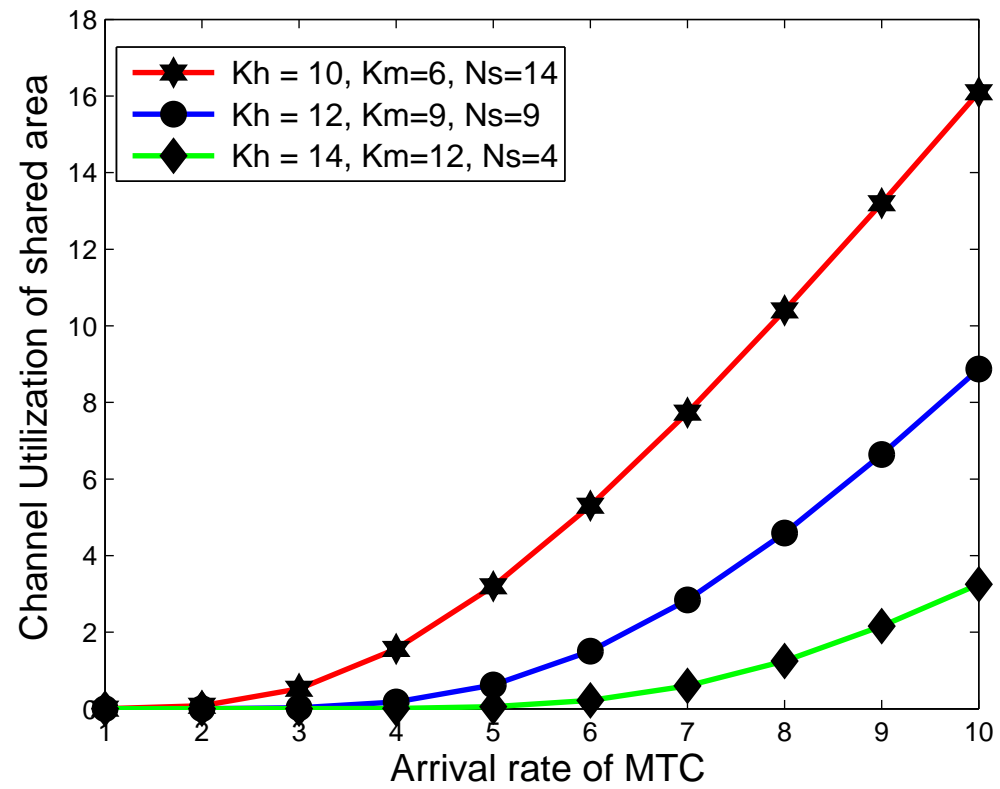

Figure 4.15: Channel Utilization of shared area vs. arrival rate of MTC with varied thresholds.

The channel utilization for the shared area is analyzed by keeping the threshold for MTC traffic constant (here $K_{m}=9$ ). The results are shown in Fig. 4.16. It can be observed that the channel utilization for the shared area increases rapidly when the arrival rate of the MTC traffic is beyond a particular level (here 4). Below that level, the channel utilization for the shared area is very low due to the fact that the MTC requests are being processed mostly by the MTC dedicated area.

Next, the channel utilization for the shared area is analyzed by keeping the threshold for HTC traffic constant (here $K_{h}=8$ ). The results are shown in Fig. 4.17. It can also be observed that the channel utilization for the shared area increases rapidly when the arrival rate of the MTC traffic is beyond a particular level (here 5). Below that level, the channel utilization for the shared area is very low due to the fact that the MTC requests are being processed mostly by the MTC dedicated area. It is also observed that low MTC thresholds tend to generate more channel utilization for the shared area.

Further, the channel utilization of shared area is analyzed when thresholds are not as- 


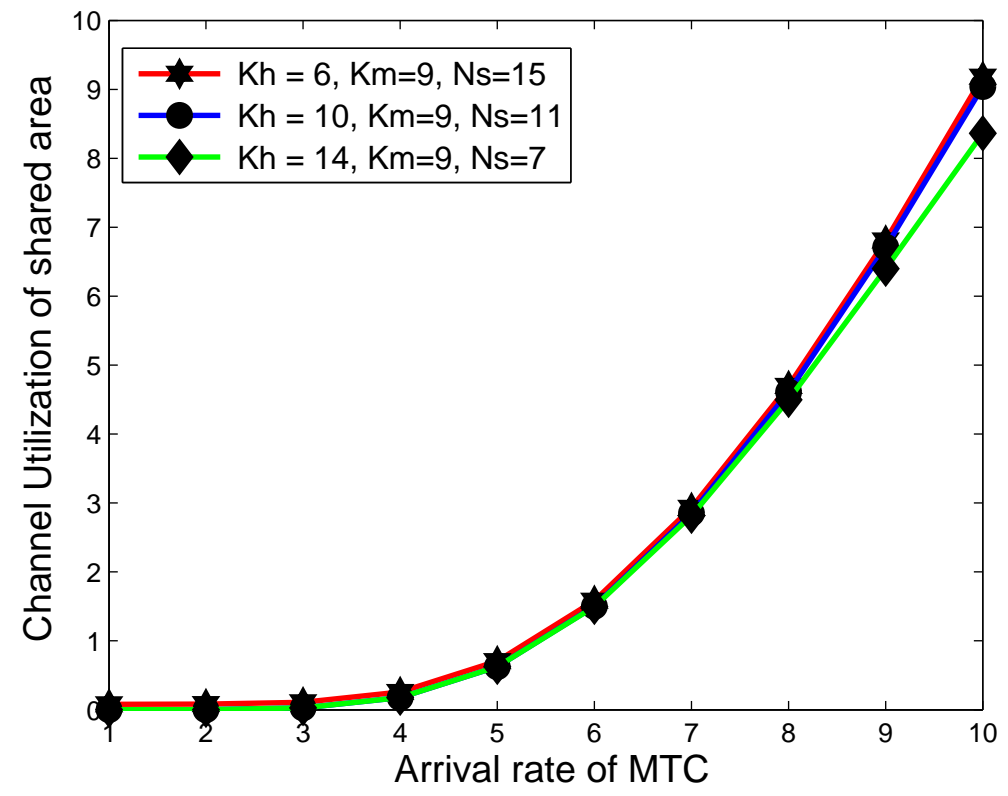

Figure 4.16: Channel Utilization of shared area vs. arrival rate of MTC with constant MTC threshold.

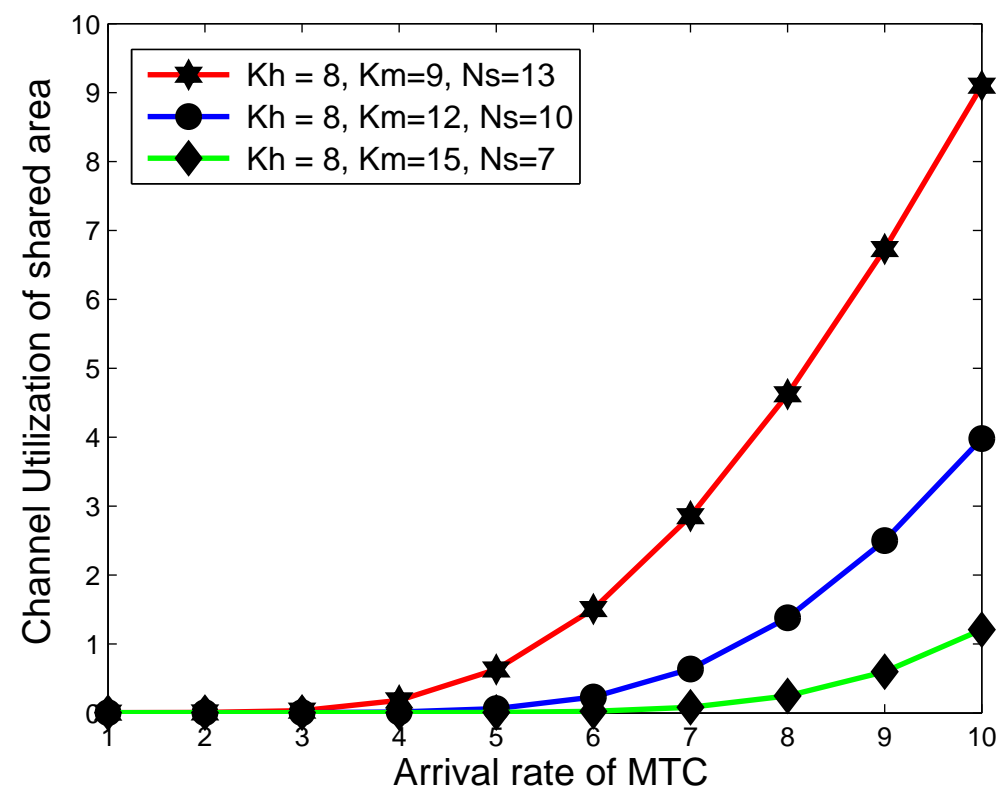

Figure 4.17: Channel Utilization of shared area vs. arrival rate of MTC with constant HTC threshold.

signed to HTC and MTC traffic. Results are shown in Fig. 4.18. When no dedicated radio channels are allocated to both MTC and HTC (e.g. $K_{h}=0$ and $K_{m}=0$ ), the channel utiliza- 


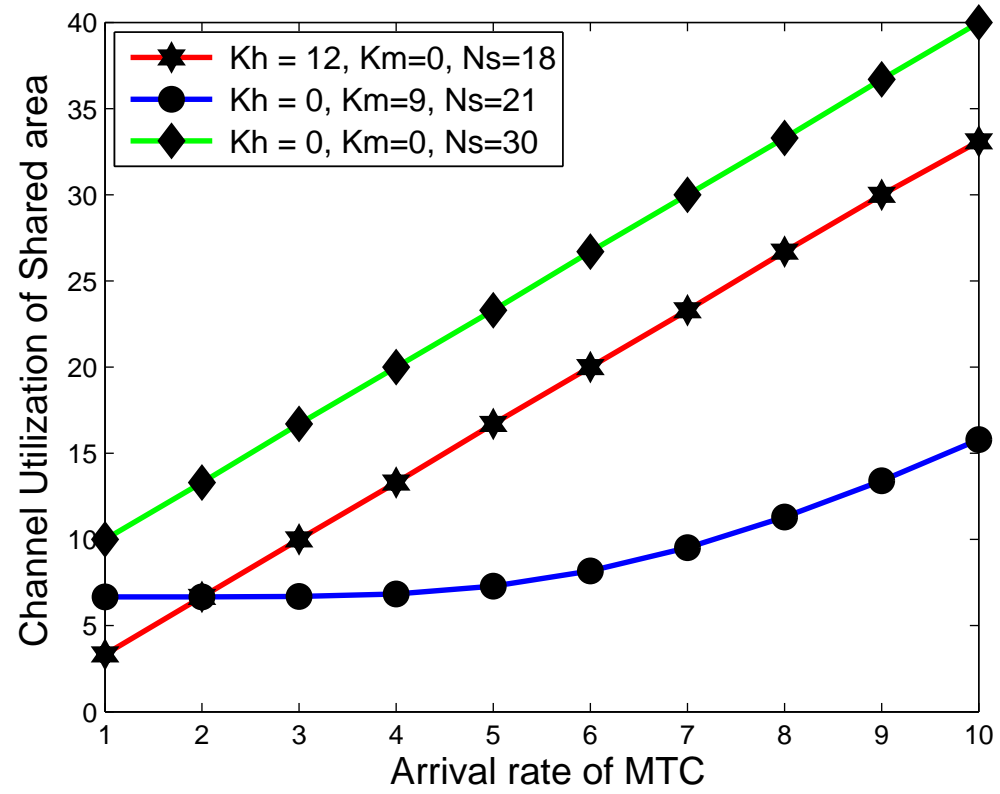

Figure 4.18: Channel Utilization of shared area vs. arrival rate of MTC without thresholds.

tion of shared area increases rapidly with increase in MTC arrival rate. This is due to the fact that both MTC and HTC requests are coming directly in shared area for processing and keeps its resources busy. Similarly when no radio channels are dedicated to MTC, all the MTC requests are served with radio channels of shared area. This increases the channel utilization of shared area with increase in arrival rate of MTC. Rate of change in shared area utilization is very less when some radio channels are dedicated to MTC (here $K_{m}=9$ ). This is because most the MTC requests are processed in MTC dedicated area and if the MTC arrival rate is low, no MTC request will use the shared area. At higher arrival rate of MTC there is a slight increase in shared area utilization as MTC dedicated area is filled out and services are now redirected to the shared area.

\subsubsection{Scenario VI: Impact of the Variation of the Arrival Rate of HTC traffic on the Channel Utilization for the Shared Area}

The arrival rate of the MTC traffic is fixed to 10 data packets per second. The arrival rate of the HTC is varied and the impact of this variation on the channel utilization for the shared 
area is studied. For this scenario, four different types of patterns are analyzed by varying the HTC and MTC threshold values. The results are captured in Fig 4.19, Fig 4.20, Fig 4.21 and Fig 4.22.

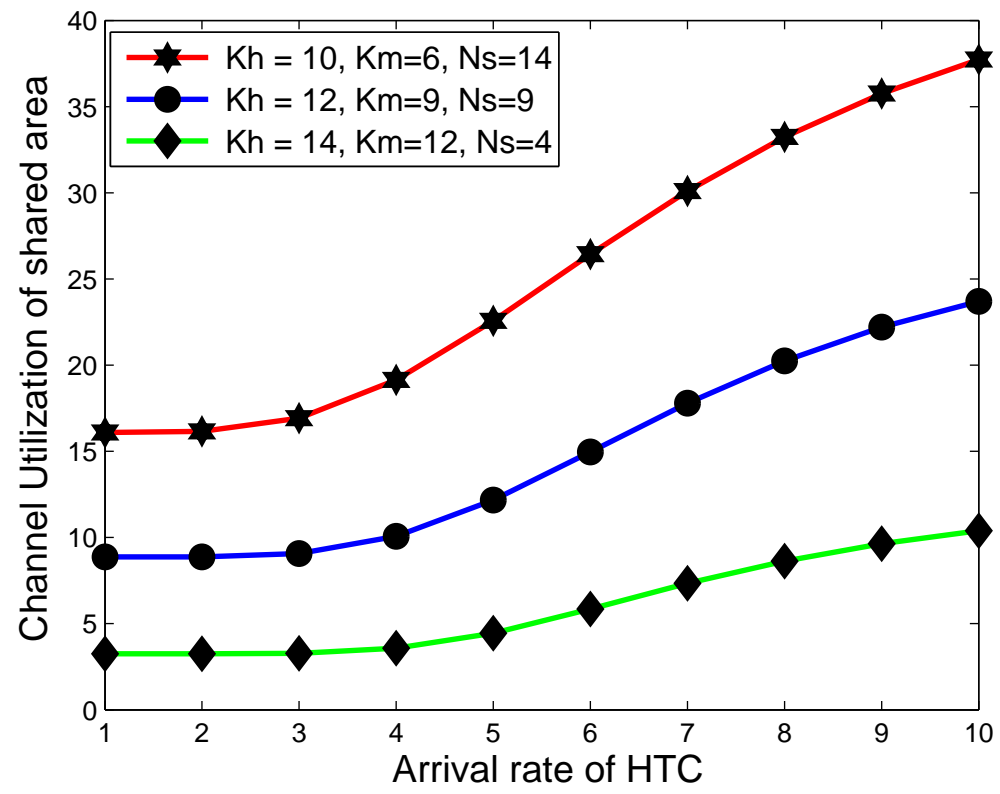

Figure 4.19: Channel Utilization of shared area vs. arrival rate of HTC with varied thresholds.

First, both HTC and MTC thresholds are varied. Fig. 4.19 shows that when the MTC and HTC thresholds are very high, there is less variation in the channel utilization of the shared area even though the HTC arrival rate is increased. This is attributed to the fact that the requests are being processed in their dedicated areas and barely use the shared area. On the other hand, when the HTC and MTC thresholds are kept at low values, the channel utilization of the shared area is increased sharply when the arrival rate of HTC traffic is high. This is due to the fact that the dedicated areas for both HTC and MTC traffic are filled out quickly when the arrival rate of HTC traffic increases, leading to more HTC requests being pushed into the shared area.

Second, the threshold on MTC traffic is kept constant. Fig. 4.20 shows that the lower the threshold on the HTC traffic, the bigger the change in the channel utilization of the 


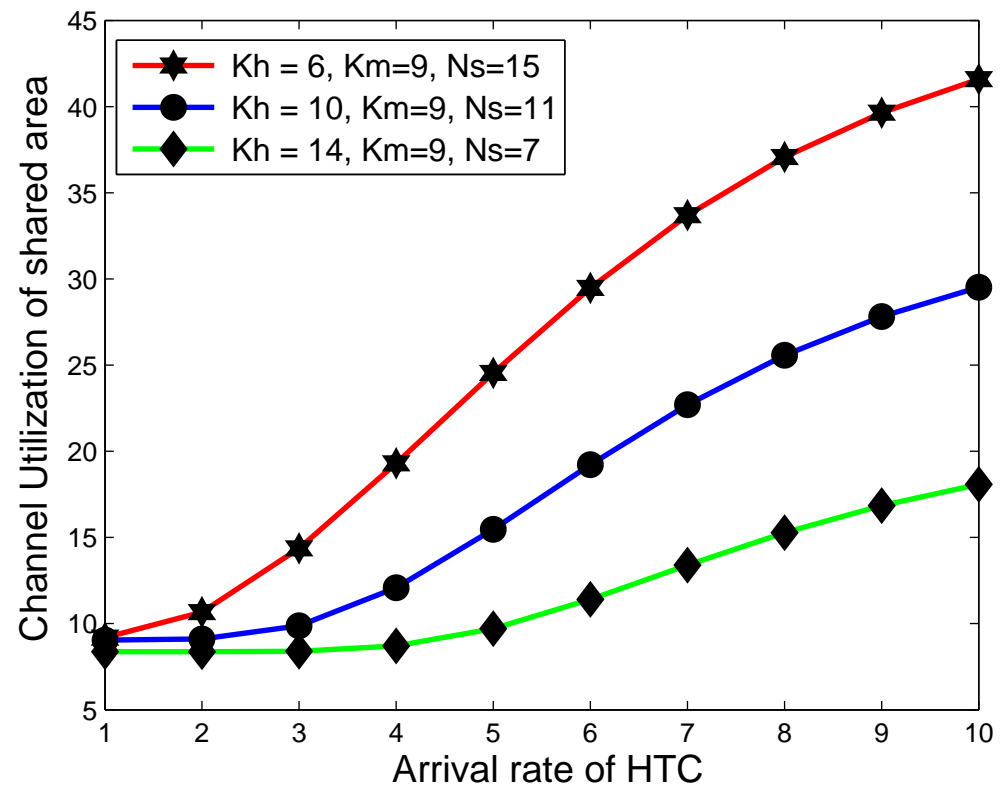

Figure 4.20: Channel Utilization of shared area vs. arrival rate of HTC with constant MTC threshold.

shared area. This is due to the fact that when the threshold of HTC traffic is low, the dedicated area for HTC traffic is filled out quickly when the arrival rate of HTC traffic increases, leading to more HTC requests being pushed into the shared area. On the other hand, when the threshold of HTC traffic is high, most of the HTC requests are served within the HTC dedicated area only until the arrival rate of HTC becomes very high, thus the channel utilization of the share area remains unchanged up to that specific high HTC arrival rate.

Third, the threshold of HTC traffic is kept constant. Fig. 4.21 shows that the channel utilization of the shared area increases with an increase in the arrival rate of HTC traffic. This is due to the fact that the threshold for HTC traffic is fixed, therefore as more HTC requests comes to the system, they need to be served with same number of resources from the HTC dedicated area. When the arrival rate of the HTC traffic becomes high, the dedicated area for the HTC traffic is filled out quickly, leading to more HTC requests being pushed into the shared area, hence increasing its utilization. It is also observed that when the threshold 


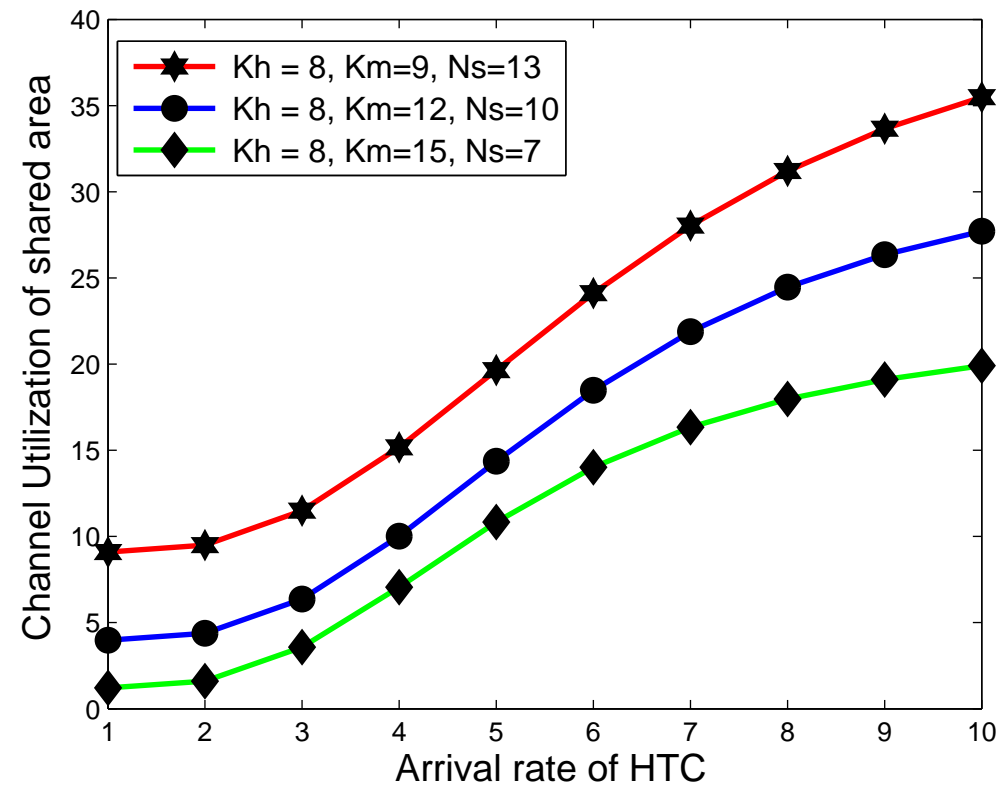

Figure 4.21: Channel Utilization of shared area vs. arrival rate of HTC with constant HTC threshold.

on the MTC traffic increases, the channel utilization of the shared area is reduced. This is attributed to the fact that the threshold on HTC traffic is kept constant, which in turn reduces the resources available in the shared area.

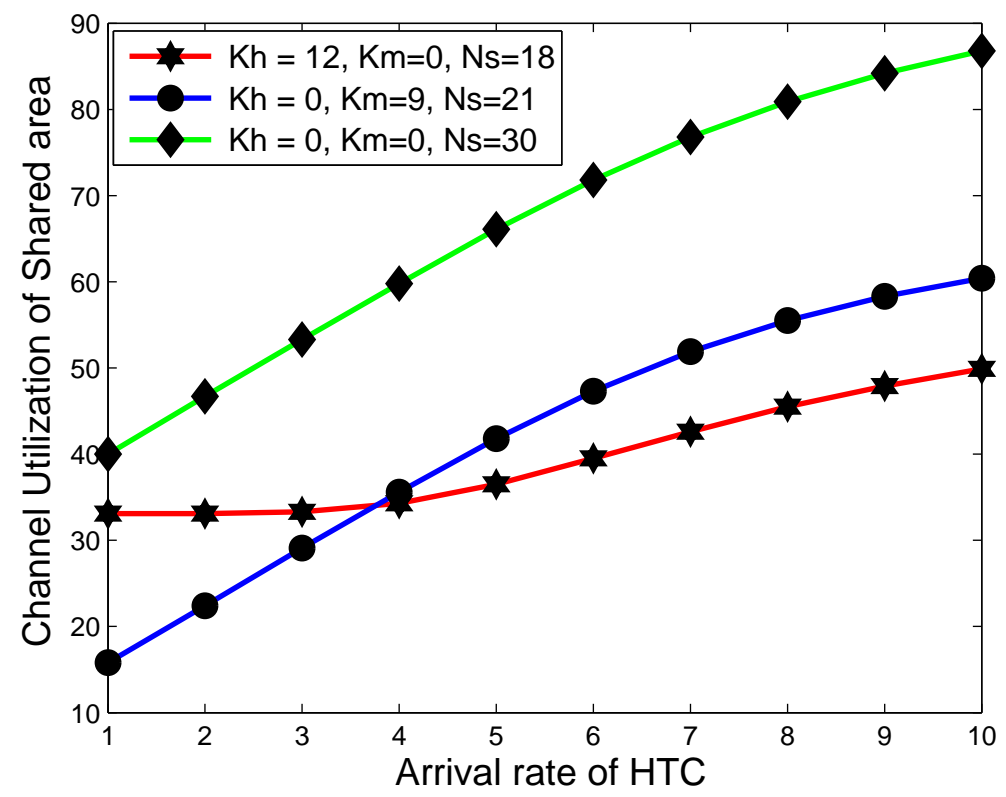

Figure 4.22: Channel Utilization of shared area vs. arrival rate of HTC without thresholds. 
Lastly, the channel utilization of shared area is analyzed when thresholds are not assigned to HTC and MTC traffic. Results are shown in Fig. 4.22. When no thresholds are assigned to both MTC and HTC traffic (e.g. $K_{h}=0$ and $K_{m}=0$ ), the channel utilization of shared area increases rapidly with increase in HTC arrival rate. This is because all the requests are directly coming in shared area for processing and keeps the resources busy. Similarly when no threshold is assigned only to HTC (e.g. $K_{h}=0$ and $K_{m}=9$ ), all the HTC requests are processed in shared area. So, with the increase in HTC arrival rate, channel utilization of shared area increases. Rate of change in shared area utilization is very less when threshold is assigned to HTC. This is due to the fact that most the HTC requests are processed in HTC dedicated area when HTC arrival rate is low. At higher arrival rate of HTC there is a slight increase in shared area utilization as services are now start getting processed in the shared area. 


\section{Chapter 5}

\section{Conclusion}

In this thesis, a novel CTMC-based RRM scheme for analyzing the performance of MTC traffic over HTC traffic in wireless communication networks is proposed. This scheme is based on the idea of partitioning the available radio channels into three separate groups: the

first dedicated to handle the HTC traffic; the second dedicated to handle the MTC traffic, and the third constituting the shared area which can be used by the HTC or MTC traffic. An analytical model has been built to investigate the effectiveness of the proposed scheme, under various scenarios. These scenarios have consisted in varying the arrival rate of the MTC traffic in the presence of HTC traffic and investigating the impact of these variations on the blocking probability and channel utilization for MTC, HTC, and shared areas. The findings are:

- With a proper selection of HTC and MTC thresholds in radio channels, the blocking probability of HTC traffic and MTC traffic can be improved. The impact of the MTC traffic on the existing HTC traffic in a cellular network can be reduced to an acceptable level by setting up some threshold limits.

- Our proposed CTMC-based model provides an effective way to control the level of resource (i.e. channel utilization) usage by the HTC and MTC traffic. This is particularly important from a network provider viewpoint since such feature can allow the 
network provider to satisfy the end userś needs by changing the threshold values on the fly. Indeed, the level of MTC traffic or service requests can be controlled or restricted by lowering the MTC threshold value and increasing the HTC threshold value.

- The channel utilization of the network is based on the arrival rate of traffic in each group. A higher arrival rate implies a better utilization of the available radio channels.

- There is a tradeoff between the channel utilization and the blocking probability as far as the traffic arrival rate is concerned. Higher arrival rates will attract more channel utilization but with the downside that the blocking probability of both the HTC and MTC traffic will increase.

- The presence of dedicated shared radio channels for HTC and MTC traffic do provide some advantages in terms of QoS of the MTC traffic without any significant degradation in the QoS of the HTC traffic.

Our proposed CTMC-based RRM scheme is based on the basic cellular system architecture so as to keep its concept and model simple and easy to understand. Several other parameters can be added to the model to make it adaptable to various types of wireless cellular networks. In future, the following studies can be pursued as extension of this work:

- Design of a systematic approach to determine the values of the thresholds in a formal way for instance, using an algorithm based on traffic load. We believe that doing so will further enhance the capability of the proposed system from a practical perspective.

- In the current CTMC-based model, to keep the system simple, we have considered only data services in MTC and HTC traffic. Voice calls and specific types of MTC applications/traffic can also be analyzed using the proposed model, in terms of blocking probability and channel utilization. This will help extending the current study by including more realistic scenarios. 
- The current CTMC-based model can be enhanced by including the queues for prioritizing the incoming traffic. This helps achieving the QoS for high demanding applications. In addition, the blocking probability of certain sets of incoming service requests can be reduced using these queues.

- To further analyze the system in a more realistic way, the packet size of the incoming service requests can be considered as additional design criterion for the model. This will lead to a possible quantification of the amount of resources needed to serve a specific incoming request on its arrival.

- The current CTMC-based model can be adjusted to be used for LTE networks in which resource blocks are allocated based on the data packet size to be transferred.

- CTMC model proposed in this thesis can also be compare against a benchmark model already proposed in the literature. A queuing model can also be integrated into the CTMC model to strengthen its practicality. 


\section{Appendix A}

\section{Pseudocode for CTMC model}

For the implementation purpose of CTMC model, we have used modesto package [28]. The pseudo-code of the CTMC model is as follows.

Input: $N_{c h}, K_{h}, K_{m}, \lambda_{H T C}, \lambda_{M T C}, \mu_{H T C}, \mu_{M T C}$

$N_{c h}=$ Total number of radio channels

$K_{h}=$ Threshold for HTC

$K_{m}=$ Threshold for MTC

$\lambda_{H T C}=$ Arrival rate of HTC service requests

$\lambda_{M T C}=$ Arrival rate of MTC service requests

$\mu_{H T C}=$ Service time of HTC service requests

$\mu_{M T C}=$ Service time of MTC service requests

$N_{s}=$ Size of shared area $\left\{\right.$ i.e $\left.N_{c h}-\left(K_{h}+K_{m}\right)\right\}$

$S_{h}=$ Number of HTC service requests.

$S_{m}=$ Number of MTC service requests.

$S_{h s}=$ Number of HTC service requests in shared area.

$S_{m s}=$ Number of MTC service requests in shared area.

for all States in $S=\left(s_{h}, s_{h s}, s_{m}, s_{m s}\right)$ do

\{ For HTC service requests\} 
\{Arrival of HTC requests in HTC dedicated area\}

if $S_{h}<K_{h}$ then

$$
S_{h}=S_{h}+1
$$

Add the transition to the process with rate $\lambda_{H T C}$.

\section{end if}

\{Departure of HTC requests from HTC dedicated area\}

if $S_{h}>0$ then

$$
S_{h}=S_{h}-1
$$

Add the transition to the process with rate $S_{h}{ }^{*} \mu_{H T C}$.

\section{end if}

\{Arrival of HTC requests in shared area\}

if $\left(S_{m s}+S_{h s}\right)<N_{s}$ then

$$
S_{h s}=S_{h s}+1
$$

Add the transition to the process with rate $\lambda_{H T C}$.

\section{end if}

\{Departure of HTC requests from shared area\}

if $S_{h s}>0$ then

$$
S_{h s}=S_{h s}-1
$$

Add the transition to the process with rate $S_{h s}{ }^{*} \mu_{H T C}$.

\section{end if}

$\{$ For MTC service requests\}

\{Arrival of MTC requests in MTC dedicated area\}

if $S_{m}<K_{m}$ then

$$
S_{m}=S_{m}+1
$$

Add the transition to the process with rate $\lambda_{M T C}$.

\section{end if}

\{Departure of MTC requests from MTC dedicated area\} 
if $S_{m}>0$ then

$$
S_{m}=S_{m}-1
$$

Add the transition to the process with rate $S_{m} * \mu_{M T C}$.

\section{end if}

\{Arrival of MTC requests in shared area\}

if $\left(S_{m s}+S_{h s}\right)<N_{s}$ then

$$
S_{m s}=S_{m s}+1
$$

Add the transition to the process with rate $\lambda_{M T C}$.

\section{end if}

\{Departure of HTC requests from shared area\}

if $S_{m s}>0$ then

$$
S_{m s}=S_{m s}-1
$$

Add the transition to the process with rate $S_{m s}{ }^{*} \mu_{M T C}$.

\section{end if}

\{Blocking probability of HTC

if $S_{h}=K_{h}$ and $N_{s}=\left(S_{h s}+S_{m s}\right)$ then

Calculates blocking probability of HTC as in equation 3.2.

\section{end if}

\{Blocking probability of MTC

if $S_{m}=K_{m}$ and $N_{s}=\left(S_{h s}+S_{m s}\right)$ then

Calculates blocking probability of MTC as in equation 3.3.

\section{end if}

\{Channel utilization of HTC

if $S_{h}>0$ or $S_{h s}>0$ then

Calculates channel utilization of HTC as in equation 3.4.

end if

$\{$ Channel utilization of MTC 
if $S_{m}>0$ or $S_{m s}>0$ then

Calculates channel utilization of MTC as in equation 3.5.

end if

\{Channel utilization of shared area\}

if $S_{h s}>0$ or $S_{m s}>0$ then

Calculates channel utilization of shared area as in equation 3.6.

end if

end for 


\section{Bibliography}

[1] "Excerpts from a conversation with gordon moore: Moores law." Intel Corporation, http://large.stanford.edu/courses/2012/ph250/lee1/docs/Excepts_A_Conversation_with_Gordon.pdf (Last visited Nov. 3, 2014), 2005.

[2] D. Minoli, Building the Internet of Things with IPv6 and MIPv6. John Wiley and Sons Inc., Hoboken, New Jersey, 2013.

[3] 3rd Generation Partnership Project, "Technical specification group services and system aspects; study on facilitating machine to machine communication in 3gpp systems; (release 8)," Technical Report 3GPP TR 22.868 V8.0.0, http://www.qtc.jp/3GPP/Specs/22868-800.pdf (Last visited Nov. 3, 2014), March 2007.

[4] T. Ryberg, "The global wireless $\mathrm{m} 2 \mathrm{~m}$ market," $\mathrm{m} 2 \mathrm{~m}$ research series, 6th ed., Berg Insight, August 2014.

[5] 3rd Generation Partnership Project, "Technical specification group services and system aspects; service requirements for machine-type communications (mtc); stage 1 (release 10)," Technical Specification 3GPP TS 22.368 V10.1.0, http://www.qtc.jp/3GPP/Specs/22368-a10.pdf (Last visited Nov. 3, 2014), June 2010.

[6] R. Liu, W. Wu, H. Zhu and D. Yang, "M2m-oriented QoS categorization in cellular network," in 7th International Conference on Wireless Communications, Networking and Mobile Computing (WiCOM), Wuhan, China, pp. 1-5, Sept 2011. 
[7] P. Makris, D. N. Skoutas, N. Nomikos, D. Vouyioukas and C. Skianis, "A context-aware backhaul management solution for combined h2h and $\mathrm{m} 2 \mathrm{~m}$ traffic," in International Conference on Computer, Information and Telecommunication Systems (CITS), May 7-8, Athens, Greece, pp. 1-5, May 2013.

[8] C-H. Wei, R-G. Cheng and S-L. Tsao "Performance analysis of group paging for machine-type communications in lte networks," IEEE Transactions on Vehicular Technology, vol. 62, pp. 3371-3382, Sept 2013.

[9] M. Jaloun and Z. Guennoun, "Wireless mobile evolution to 4g network," Wireless Sensor Networks, vol. 2, no. 4, pp. 309-317, 2010.

[10] "1946: First mobile telephone call." AT\&T Intellectual Property, http://www.corp.att.com/attlabs/reputation/timeline/46mobile.htm (Last visited Nov. 3, 2014).

[11] "A legacy of innovation: Timeline of motorola history since 1928." Motorola Solutions; $\quad$ http://www.motorolasolutions.com/USEN/About/Company+Overview/History/Timeline (Last visited Nov. 3, 2014).

[12] 3rd Generation Partnership Project, "Digital cellular telecommunications system (phase 2+); universal mobile telecommunications system (umts); lte; quality of service (QoS) concept and architecture; release 11)," Technical Specification 3GPP TS 23.107 version 11.0 .0 http : //www.etsi.org/deliver/etsi $i_{t}$ /123100_123199/123107/11.00.00_60/ts_123107v110000p.pdf (Last visited Nov. 3, 2014), 2012.

[13] E. Mutafungwa, Applying MTC and Femtocell Technologies to the Continua Health Reference Architecture. Springer Verlag, 2011.

[14] "Gsma." http://www.gsma.com/newsroom/gsma-predicts-250-million-m2m (Last visited Nov. 3, 2014). 
[15] T. Taleb and A. Kunz, "Machine type communications in 3gpp networks: potential, challenges, and solutions," IEEE Communications Magazine, vol. 50, pp. 178-184, March 2012.

[16] 3rd Generation Partnership Project, "Technical specification group services and system aspects; system improvements for machine-type communications (mtc) (release 11)," Technical Report 3GPP TR 23.888 V11.0.0, http://www.qtc.jp/3GPP/Specs/23888b00.pdf (Last visited Nov. 3, 2014), Sept 2012.

[17] E. Hossain, D. Niyato and Z. Han, Dynamic Spectrum Access and Management in Cognitive Radio Networks. Cambridge University Press, New York, 2009.

[18] S-Y. Lien, K-C. Chen and Y. Lin, "Toward ubiquitous massive accesses in 3gpp machine to machine communications," IEEE Communication Magazine, vol. 49, pp. 66-74, April 2011.

[19] I. Bang, K-S. Ko and D-K. Sung , "A user-pairing based resource allocation scheme for a large number of devices in $\mathrm{m} 2 \mathrm{~m}$ communications," in IEEE 24th International Symposium on Personal Indoor and Mobile Radio Communications (PIMRC), pp. 15541558, Sept 2013.

[20] A. Aijaz and A. H. Aghvami, "On radio resource allocation in lte networks with machineto-machine communications," in IEEE r7th Vehicular Technology Conference (VTC Spring), pp. 1-5, June 2013.

[21] K. Zheng, F. Hu, W. Wang, W. Xiang and M. DDohler, "Radio resource allocation in lte advanced cellular networks with m2m communications," IEEE Communications Magazine, vol. 50, pp. 184-192, July 2012.

[22] T. Potsch, S. N. Khan Marwat, Y. Zak and C. Gorg, "Influence of future m2m communication on the lte system," in 6th Joint IFIP Wireless and Mobile Networking Conference (WMNC), pp. 1-4, April 2013. 
[23] M. K. Giluka, N. Sharath Kumar, N. Nitish Rajoria and B. R. Tamma, "Class based priority scheduling to support machine to machine communications in lte systems," in Twentieth National Conference on Communications (NCC 2014), IIT Kanpur, India, pp. 1-6, Feb 2014.

[24] Y-H. Hsu, K. Wang and Y.-C. Tseng, "Enhanced cooperative access class barring and traffic adaptive radio resource management for $\mathrm{m} 2 \mathrm{~m}$ communications over lte-a," in Asia-Pacific Signal and Information Processing Association Annual Summit and Conference (APSIPA 2013), Kaohsiung, Taiwan, R.O.C, pp. 1-6, Oct 2013.

[25] T-M. Lin, C-H. Lee, J.-P. Cheng and W.-T. Chen, "Prada: Prioritized random access with dynamic access barring for mtc in 3gpp lte-a networks," IEEE Transactions on Vehicular Technology, vol. 63, pp. 2467-2472, June 2014.

[26] K-D. Lee, S. Kim and B. Yi, "Throughput comparison of random access methods for m2m service over lte networks," in IEEE GLOBECOM Workshops, Houston, TX, USA, pp. 373-377, Dec 2011.

[27] C. Ide, D. Bjoern and C. Wietfeld, "Performance of channel-aware m2m communications based on lte network measurements," in IEEE 24th International Symposium on Personal Indoor and Mobile Radio Communications (PIMRC), London, UK, pp. 16141618, Sept 2013.

[28] G. Carvalho, V. S. Martins, C. Frances, J. Costa and S. Carvalho, "Performance analysis of multi-service wireless network: An approach integrating CAC, scheduling, and buffer management," Computers and Electrical Engineering, vol. 34, pp. 346-356, 2008. 